\title{
On the use of Saint Venant equations to simulate the spreading of a granular mass
}

\author{
A. Mangeney-Castelnau, ${ }^{1,2}$ F. Bouchut, ${ }^{3}$ J. P. Vilotte, ${ }^{1}$ E. Lajeunesse, ${ }^{2,4}$ A. Aubertin,,${ }^{1,2}$ \\ and M. Pirulli ${ }^{1,2,5}$
}

Received 4 May 2004; revised 9 March 2005; accepted 11 April 2005; published 16 September 2005.

[1] Cliff collapse is an active geomorphological process acting at the surface of the Earth and telluric planets. Recent laboratory studies have investigated the collapse of an initially cylindrical granular mass along a rough horizontal plane for different initial aspect ratios $a=H_{i} / R_{i}$, where $H_{i}$ and $R_{i}$ are the initial height and radius, respectively. A numerical simulation of these experiments is performed using a minimal depth-integrated model based on a long-wave approximation. A dimensional analysis of the equations shows that such a model exhibits the scaling laws observed experimentally. Generic solutions are independent of gravity and depend only on the initial aspect ratio $a$ and an effective friction angle. In terms of dynamics, the numerical simulations are consistent with the experiments for $a \leq 1$. The experimentally observed saturation of the final height of the deposit, when normalized with respect to the initial radius of the cylinder, is accurately reproduced numerically. Analysis of the results sheds light on the correlation between the area overrun by the granular mass and its initial potential energy. The extent of the deposit, the final height, and the arrest time of the front can be directly estimated from the "generic solution" of the model for terrestrial and extraterrestrial avalanches. The effective friction, a parameter classically used to describe the mobility of gravitational flows, is shown to depend on the initial aspect ratio $a$. This dependence should be taken into account when interpreting the high mobility of large volume events.

Citation: Mangeney-Castelnau, A., F. Bouchut, J. P. Vilotte, E. Lajeunesse, A. Aubertin, and M. Pirulli (2005), On the use of Saint Venant equations to simulate the spreading of a granular mass, J. Geophys. Res., 110, B09103, doi:10.1029/2004JB003161.

\section{Introduction}

[2] Dense gravitational flows, triggered by large mass destabilization, are active processes that participate in the evolution of the surface of the Earth and other telluric planets. They also represent natural hazards that are today a threat to many populations and industrial infrastructures. Investigation of dense gravitational flows has a long history in geology and geomechanics. Extensive field and remote sensing observations have led to a comprehensive description of their morphogenesis. However, field studies are difficult and the underlying physics and environmental processes of dense gravitational flows remain poorly understood, partly due to the wide range of physical regimes, as well as different space and timescales, involved in

\footnotetext{
${ }^{1}$ Département de Sismologie, Institut de Physique du Globe de Paris, Paris, France.

${ }^{2}$ Groupe de Recherches sur 1'Erosion et les Eaux Continentales, Institut de Physique du Globe de Paris, Paris, France.

${ }^{3}$ Département de Mathématique et Applications, Ecole Normale Supérieure et CNRS, Paris, France.

${ }^{4}$ Laboratoire de Dynamique des Systèmes Géologiques, Institut de Physique du Globe de Paris, Paris, France.

${ }^{5}$ Politecnico, Torino, Italy.
}

Copyright 2005 by the American Geophysical Union. 0148-0227/05/2004JB003161\$09.00 phenomena ranging from cliff collapse to rapid debris flows.

[3] The underlying physical principles driving dense gravitational flows have been recently investigated through laboratory experiments and conceptual dense granular models. Clearly, a large number of time and space scales are involved, differing by many order of magnitudes. An unresolved issue is the continuum description of dense gravitational flows, from laboratory scale to the large scales of complex geological flows and incorporating the relevant environmental parameters.

[4] Dense dry granular flows along inclined planes have been shown to exhibit a number of characteristics of natural gravitational flows [e.g., Felix and Thomas, 2004; Iverson, 1997; Denlinger and Iverson, 2001] and have been thoroughly investigated [Gray et al., 1999; Pouliquen, 1999; Pouliquen and Forterre, 2002]. Simple continuum hydrodynamic models, based on the long-wave approximation (LWA) [Savage and Hutter, 1989] and Saint Venant equations, have been shown to reproduce basic features of both experimental dense granular flows along inclined planes and geological flows along real topographies [e.g., Denlinger and Iverson, 2001; Naaim et al., 1997; Pastor et al., 2002; Pouliquen and Forterre, 2002; MangeneyCastelnau et al., 2003; Pitman et al., 2003; Denlinger and Iverson, 2004; Iverson et al., 2004, Sheridan et al., 2005]. The LWA is a natural approximation for these flow regimes 
given the low aspect ratio between the vertical (the avalanche thickness) and horizontal (the avalanche runout) length scales and the small ratio of the surface topography amplitude to the typical vertical length scale. Continuum models are expressed in terms of the change of the vertically averaged velocity field and of the associated vertical length scale $h$, i.e., the avalanche thickness, and describe hydrostatic imbalance [Mangeney-Castelnau et al., 2003] or nonhydrostatic plasticity and friction effects [Savage and Hutter, 1989]. The models assume an averaged friction dissipation described phenomenologically in the framework of Coulomb's friction with a constant [e.g., Hutter et al., 1995; Naaim et al., 1997] or velocity- and height-dependent [Pouliquen, 1999; Douady et al., 1999] friction coefficient.

[5] Although quite important in terms of risk assessment, cliff collapse, which involves mechanisms of mass spreading quite different from classical flows, has been the subject of fewer studies. Recently, laboratory experiments involving collapse and spreading of a granular mass have been performed by Lajeunesse et al. [2004] and Lube et al. [2004]. In both experiments a granular mass of cylindrical shape was suddenly released on a quasi-horizontal surface. The main conclusion is that the spreading and final deposit are controlled by the aspect ratio of the initial mass. Depending on the initial aspect ratio $a$, two different flow regimes are identified. Empirical relations were derived relating the runout distance and the height of the deposit to the initial aspect ratio $a$ of the cylindrical mass.

[6] The aim of this paper is to investigate these experiments numerically. Two approaches are possible. Discrete element methods make use of an explicit description, at the particle scale, of the behavior of the granular mass and make it possible to take into account nonhomogeneous deformations and potential localization patterns during the collapse [e.g., Müller, 1995; Staron and Hinch, 2004]. Although this approach provides insight into the mechanical behavior of the granular mass during spreading, its application to large extended systems is difficult for computational reasons. A continuum-like approach is therefore needed. As previously mentioned, continuum hydrodynamic models based on the LWA and Saint Venant equations have been widely used to simulate granular flows along inclined planes or topography. In this case, the LWA is a natural approximation due to the small aspect ratio of the flow $\delta=H / L$, where $H$ is the height of the flowing material and $L$ the running distance. Obviously, such a geometrically based argument is not necessarily relevant in collapse experiments. However, note that the LWA is a kinematic approximation based on the existence of a spatial scale, e.g., the vertical scale, below which rapid fluctuations of the velocity field can be smoothed out through a vertical averaging, and small vertical accelerations. Therefore for a leading order approximation, classical LWA gives flow models characterized by a weak hydrostatic imbalance on the vertically averaged horizontal velocity of an incompressible fluid possessing a free surface and moving under the force of gravity. Although simple geometrical scaling arguments cannot be used for mass collapse experiments, it is hard to know a priori if LWA can or cannot be applied to describe the spreading. In fact, the initial geometry is not preserved during the spreading, leading to a lower aspect ratio geometry. Moreover, the flow, at a distance $r>R_{i}$ from the center of the mass, where $R_{i}$ is the initial radius of the mass, bears a strong resemblance to thin skin flow and at a distance $r<R_{i}$ the flowing region is expected to be located near the surface.

[7] We therefore investigated numerically the mass collapse experiments using a LWA approximation. The validity domains of such an assumption will be analyzed by direct comparison with the experimental results, both in terms of flow dynamics and the final shape of the deposit. In these situations, direct investigation of the scaling of the governing equations may provide an explanation of the key role played by the initial aspect ratio, as suggested by the experiments. Moreover, numerical experiments of the spreading make it possible to explore numerically the sensitivity of the results to various parameters, such as the initial shape of the released mass, and to extend this exploration to the larger volumes involved in real events.

[8] While this paper was being finalized, several authors published new results for numerical studies of this phenomenon. Where comparison is possible, the results of the analytical and numerical approach performed by Kerswell [2005], also based on Saint Venant equations with Coulomb friction, appear consistent with those reported here. This study shows that the spreading of granular columns are purely extensional. Only one of the Earth pressure coefficients reflecting whether the material is locally extending or contracting [Savage and Hutter, 1989] is then relevant and this can easily be scaled out of the problem. Furthermore the discrete element simulation performed by L. Staron and E. J. Hinch (Study of the collapse of granular columns using DEM numerical simulation, submitted to Journal of Fluid Mechancis, 2005) provides insight into the heterogeneous behavior of granular collapse.

[9] We first briefly describe, in section 2, the experimental results used for this study. Then after a short presentation of the model, a scaling analysis of the LWA model is performed in section 3 , providing new insight into the experimental observations. A qualitative comparison between the experimental and numerical results is presented in section 4 where the accuracy and the intrinsic limitations of the LWA approach are discussed. In section 5, the dependence of the deposit morphology on the control parameters is investigated numerically and empirical scaling relations, similar to those derived experimentally, are presented. Finally, in section 6, implications of the simulations to the spreading of large volumes are discussed with reference to geological observations.

\section{Experimental Setup and Results}

[10] We will first briefly outline the experimental setup and the main results of Lajeunesse et al. [2004]. The experimental setup is made of a cylinder, with an inner radius $R_{i}$, lying on a horizontal $60 \mathrm{~cm} \times 60 \mathrm{~cm}$ plane. The cylinder is partially filled with glass beads, of diameter $d=$ $350 \pm 50 \mu \mathrm{m}$, so as to form a column of radius $R_{i}$ and height $H_{i}$ with mass $M$. The angle of repose $\theta_{r} \sim 21^{\circ}$ and the angle of avalanche $\theta_{a} \sim 29^{\circ}$ of the granular material are approximated from the estimation of the slope of the granular pile built by slowly pouring the beads from a small height [Lajeunesse et al., 2004]. The angle of avalanche corresponds to the limiting angle of stability of the pile, while the 
angle of repose corresponds to the angle of the pile after avalanche. The density of the glass beads is $\rho_{0}=2500 \mathrm{~kg}$ $\mathrm{m}^{-3}$ and the mean density of packing of the granular column is roughly estimated as $\phi \sim 0.62-0.65$, a typical value for dense initial heaps [Daerr and Douady, 1999].

[11] The granular material enclosed inside the tube is suddenly allowed to spread, by removing the tube, on the horizontal plane. When released, the granular mass collapses and spreads until it comes to rest and forms a deposit, as shown in Figure 1, where the same experiment is performed numerically. The change of the granular mass with time is monitored by a high-speed camera. Profiles $h(r, t)$ of the granular mass are measured as a function of time $t$ along with the radial distance $r$ from the axis of symmetry of the granular mass. More details are given by Lajeunesse et al. [2004]. Among other parameters, experiments were performed for different initial masses $M$ and different initial aspect ratios $a=H_{i} / R_{i}$ of the granular column. The investigated range of aspect ratios varies from $a \sim 0.2$ to $a \sim 20$. The accuracy of the experimental measurements decreases as the initial aspect ratio increases. For such initial shapes, motion occurs before the tube has been completely removed.

[12] Lajeunesse et al. [2004] showed that the spreading of the granular mass can be classified into two different flow regimes depending on the initial aspect ratio $a$ of the granular mass. In the first regime, for $a<3$, the spreading of the granular mass is accommodated by surface avalanches along the flanks and only a fraction of the initial gravitational energy is actually dissipated. The morphology of the deposit makes it possible to subdivide this regime. For $a<0.74$, the flow ends before complete consumption of the undisturbed central region by the avalanche: the characteristic shape of the final deposit is a "truncated cone" of height $H_{f}=H_{i}$ with an angle close to the angle of repose of the beads $\theta_{r}=21^{\circ}$. For $a>0.74$, the avalanche completely destroys the central region. The characteristic shape of the deposit is a cone of height $H_{f}<H_{i}$. In the second regime, for $a>3$, the initial gravitational energy is almost totally dissipated by the flow. While for $0.74<a<3$, the final deposit is characterized all along the profile by a positive curvature, for $a>3$, it is characterized by an inflection point, i.e., the transition between an outer region of negative curvature and a central region of positive curvature, leading at the end to a "sombrero" shape with a large and almost flat outer region and a steep central cone. Experiments suggest that the spreading results from surface flows involving a significant number of glass beads. Furthermore, the shape of the deposit, normalized by dividing by the initial radius, as a function of the normalized radius $h\left(r / R_{i}\right) /$ $R_{i}$ is shown to be independent of the mass $M$ but to vary with the aspect ratio $a$. For $a>0.74, H_{f} / R_{i}$ is observed to saturate roughly approaching a value of the order of 0.74 . This value has been associated with the angle of a failure surface within the granular column occurring upon removal of the tube $\delta_{c} \sim \tan ^{-1}(0.74) \sim 36,5^{\circ}$ [Lajeunesse et al., 2004]. The rescaled front velocity and the amount of energy dissipated during the flow also appear to be independent of the mass but to vary with $a$. The results are shown to be independent of the nature of the bed and of the bead size.

[13] In the following, we perform the numerical simulation of these experiments using the LWA and a depth- integrated model. We investigate to what extent such a model is able to reproduce the scaling laws observed experimentally and the averaged spreading behavior.

\section{Mathematical and Numerical Model}

\subsection{Equations}

[14] A long-wave approximation is classical for fluid dynamics problems when it is important to separate largescale motion from motion on smaller time and length scales. It is based on an asymptotic expansion in powers of one or more small parameters, one being typically a length scale below which rapid fluctuations of the velocity field can be smoothed out. The Savage-Hutter empirical model for granular flows was derived using such an approximation. Such a model describes long-time effects of slowly varying bottom topography, and of weak hydrostatic imbalance on the vertically averaged horizontal velocity of an incompressible fluid, with a free surface, that moves under the force of gravity with friction dissipation [e.g., Gray et al., 1999; Pouliquen and Forterre, 2002; Mangeney-Castelnau et al., 2003].

[15] Assuming the vertical velocity to be smaller than the characteristic tangential velocity, together with a length scale for the vertical fluctuations of the velocity smaller than that for the horizontal fluctuations of the same order, we shall consider here a minimal model derived from a purely inviscid incompressible fluid together with phenomenological friction dissipation along planes parallel to the bottom topography. The reduced governing equations are then obtained by vertically averaging the equations and by using a leading order approximation neglecting the Lagrangian vertical acceleration. For a flat bottom, the resulting equations are

$$
\begin{gathered}
\frac{\partial h}{\partial t}+\operatorname{div}(h \mathbf{u})=0 \\
\frac{\partial}{\partial t}(h u)+\frac{\partial}{\partial x}\left(h u^{2}\right)+\frac{\partial}{\partial y}(h u v)=-\frac{\partial}{\partial x}\left(g \frac{h^{2}}{2}\right)+\frac{1}{\rho} T_{x}, \\
\frac{\partial}{\partial t}(h v)+\frac{\partial}{\partial x}(h u v)+\frac{\partial}{\partial y}\left(h v^{2}\right)=-\frac{\partial}{\partial y}\left(g \frac{h^{2}}{2}\right)+\frac{1}{\rho} T_{y},
\end{gathered}
$$

where $\mathbf{u}=(u, v)$ denotes the depth-averaged horizontal flow velocity in the horizontal-vertical Cartesian reference frame $(x, y, z), h$ is the free upper surface, $\rho$ is the mass density, and $g$ is acceleration due to gravity. These equations model the hydrostatic imbalance in presence of an averaged friction force $\mathbf{T}_{t}=\left(T_{x}, T_{y}\right)$, parallel to the horizontal plane, and which is an effective approximation of the friction effects arising both at the bottom and within the bulk due to differential motion between flowing layers parallel to the bottom surface. See [Pouliquen, 1999] for a similar formulation.

[16] Note that more sophisticated models of friction dissipation have been proposed by Savage and Hutter [1989], Iverson and Denlinger [2001], and Iverson et al. [2004], who also take into account friction dissipation arising from deformation within the flowing layers parallel 


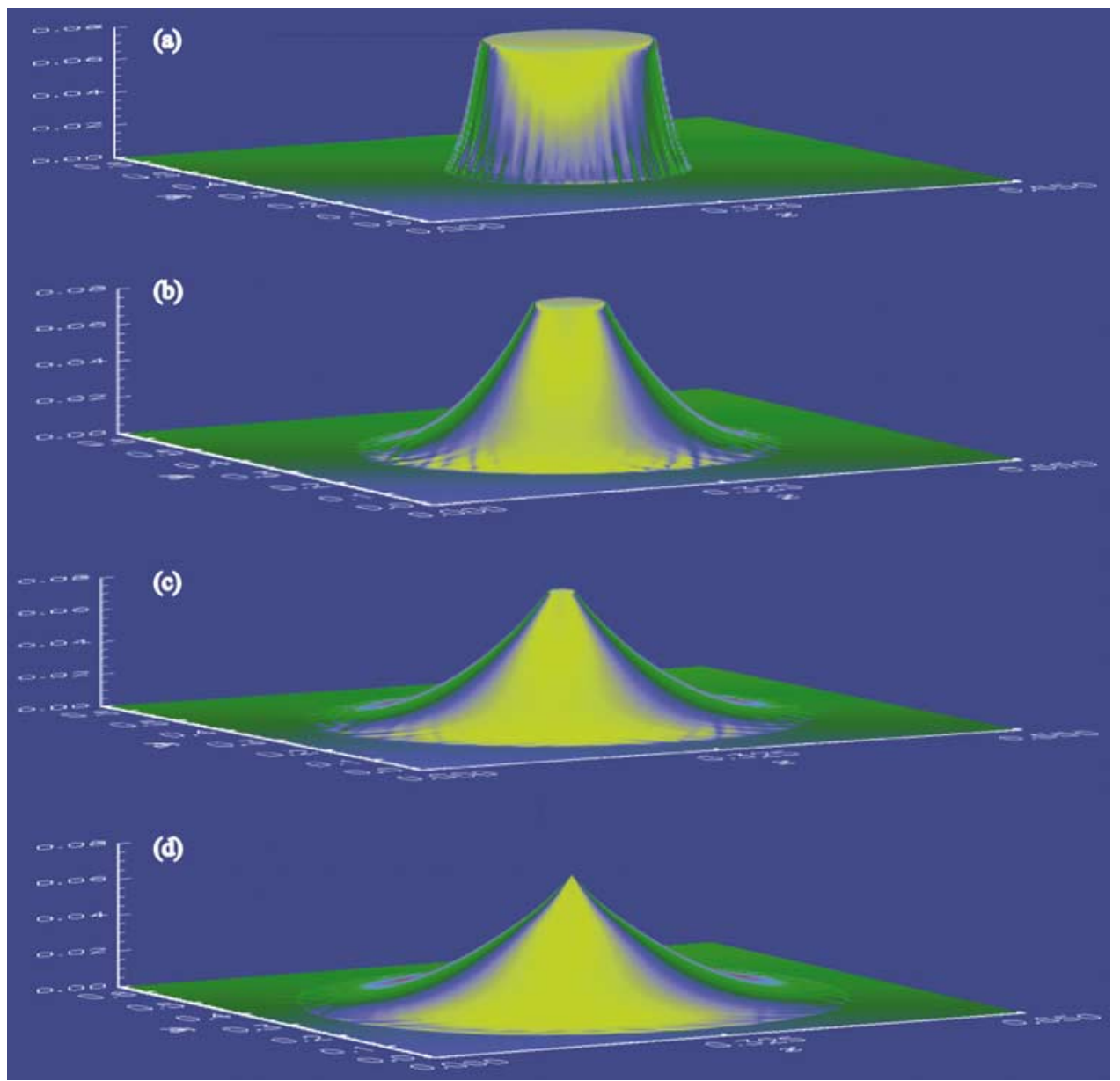

Figure 1. Numerical simulation and corresponding experimental results at time (a) and (e) $t=15 \mathrm{~ms}$, (b) and (f) $t=75 \mathrm{~ms}$, (c) and (g) $t=120 \mathrm{~ms}$, and (d) and (h) at the time of deposit, showing the spreading of an initially cylindrical granular mass with aspect ratio $a=0.8$.

to the basal plane. As a result, the averaged total spherical stress becomes anisotropic and the gravity wave celerity $c=$ $\sqrt{g h}$ must be rescaled by a factor $\|k\|^{1 / 2}$, where $k$ depends phenomenologically on both a basal friction angle and an internal friction angle that are to be determined independently experimentally. The effective parameter may depend on the sign of the divergence of the averaged tangential velocity field. The effect of $k$ would be to change the lateral stresses. This effect would be increased with increasing values of the aspect ratio $a$ due to increasing value of the pressure gradient (i.e., the surface gradient). Such anisotropy could have some influence on the spreading when the initial aspect ratio of the granular mass increases; however, such an analysis is beyond the scope of this paper. In these phenomenological models, the internal friction effect is an additional vertically averaged friction effect that can be related to potential nonhomogeneities of the tangential averaged kinematic field. However, the key problem in spreading experiments is in fact potential vertical nonhomogeneities of the deformation during spreading. Note that actually no physically or experimentally based threedimensional (3-D) continuum model exists for the flow of granular materials. It seems quite reasonable to start with minimal continuum models and to investigate qualitatively these minimal models by direct comparison with exper- imental experiments. Moreover, various discrete element simulations [Ertas et al., 2001; Volfson et al., 2003] tend to support stress isotropy during granular flows, as well as previous studies that directly compare granular flow laboratory experiments along rough inclined planes with LWA numerical simulations [Pouliquen and Forterre, 2002].

[17] The friction force has a direction opposite to the averaged tangential velocity field and when flowing, the amplitude of the friction force is governed by a friction coefficient and the total overall pressure, i.e., $\mu=\left\|\mathbf{T}_{t}\right\| / \rho g h$, where $\mu=\tan \delta$ with $\delta$ the friction angle. The transition between static and fluid behavior is simply modeled here using a Coulomb type transition [Mangeney-Castelnau et al., 2003], i.e.,

$$
\begin{aligned}
& \left\|\mathbf{T}_{t}\right\| \geq \sigma_{c} \Rightarrow \mathbf{T}_{t}=-\mu \rho g h \frac{\mathbf{u}}{\|\mathbf{u}\|} \\
& \left\|\mathbf{T}_{t}\right\|<\sigma_{c} \Rightarrow \mathbf{u}=0,
\end{aligned}
$$

where $\sigma_{c}=\mu \rho g h$.

[18] Note that granular flow laboratory experiments [Pouliquen, 1999] on inclined planes suggest, beside a domain of high inclination angles, height- and velocitydependent friction. Building on the idea of investigating a 


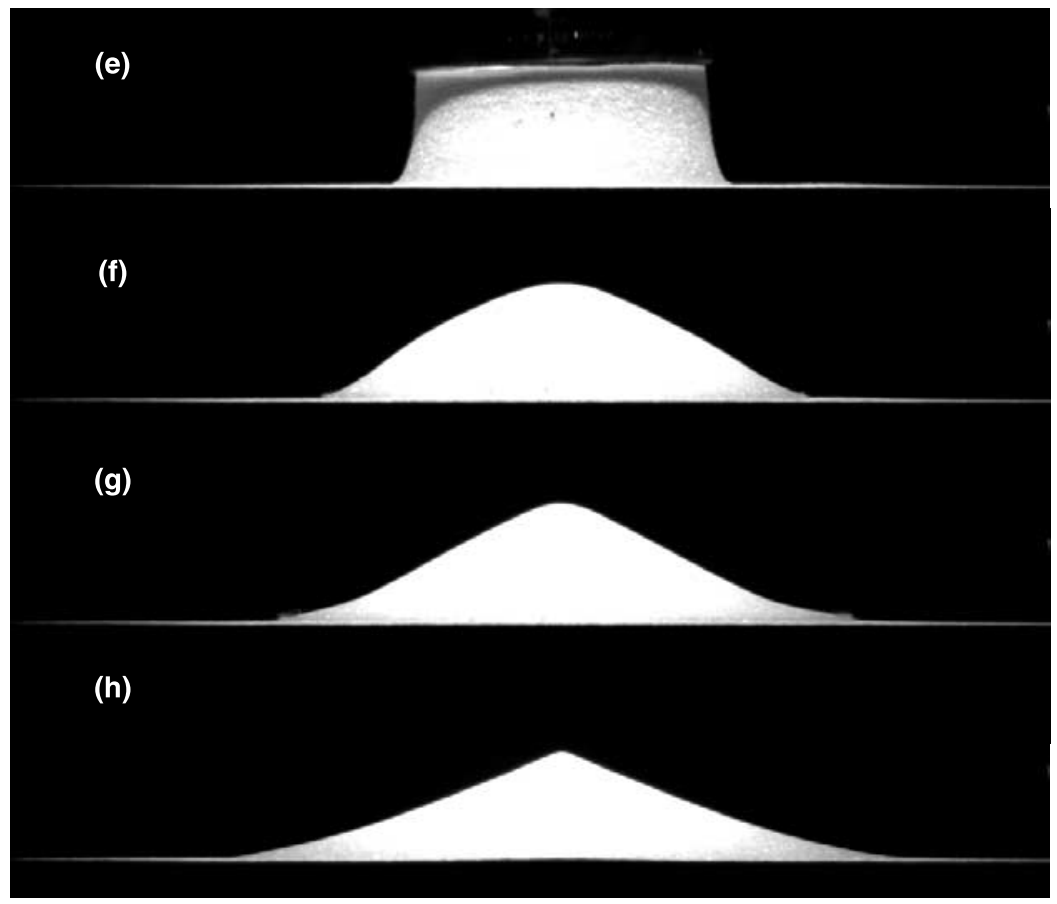

Figure 1. (continued)

minimal model for the simulation of the spreading experiments, a constant friction approximation is retained here, and the effective friction angle $\delta$ can be identified as the limiting angle of stability of a cone on a rough horizontal plane. For the minimal model considered here, the stability limit of a cone initially at rest is simply given by

$$
\begin{gathered}
\|\boldsymbol{\nabla} h\| \leq \tan \delta \Rightarrow \mathbf{u}=0 \\
\|\boldsymbol{\nabla} h\|>\tan \delta \Rightarrow \text { spreading, }
\end{gathered}
$$

which leads to identification of the effective friction angle $\delta$ of the model as the limiting angle of stability of the "numerical cone." A cone with a slope smaller than $\tan \delta$ will remain at rest while for a larger slope, flow occurs with a spreading of the cone along the plane until the dynamic forces become less or equal to the Coulomb threshold, leading to a new static equilibrium. The effective angle $\delta$ could be directly identify by experiments involving the release of a conical mass, unfortunately such experiments were not available at the time of this study.

\subsection{Scaling of the Equations}

[19] One of the main results of the spreading experiments of Lajeunesse et al. [2004] and Lube et al. [2004] was that the height of the final deposit, when normalized by division by the initial radius, obeys a scaling relationship with the normalized final radius of the deposit, i.e., $h\left(r / R_{i}\right) / R_{i}$, which is shown to be mass $M$ independent and to depend only on the initial aspect ratio $a$ of the cylinder. Such scaling law is intrinsically contained in the equations of our minimal model (1)-(3) together with the assumed Coulomb friction law (4). With appropriate dimensionless analysis, the generic form of the solution can be shown to be independent of the mass and in addition acceleration due to gravity.
Among others, let us consider the following dimensionless variables: horizontal and vertical distances are scaled by $R_{i}$ and $H_{i}$, respectively; the mean tangential velocity is scaled with the gravity wave celerity $V=\sqrt{g H_{i}}$; time is scaled by $T=\sqrt{\frac{R_{i}}{g}}$. Introducing the dimensionless variables,

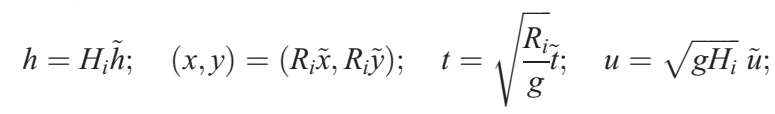

in equations (1), (2), (3), and (4) a new set of dimensionless equations is obtained

$$
\begin{gathered}
\frac{\partial \tilde{h}}{\partial \tilde{t}}+\sqrt{a} \frac{\partial}{\partial \tilde{x}}(\tilde{h} \tilde{u})+\sqrt{a} \frac{\partial}{\partial \tilde{y}}(\tilde{h} \tilde{v})=0 \\
\sqrt{a} \frac{\partial}{\partial \tilde{t}}(\tilde{h} \tilde{u})+a \frac{\partial}{\partial \tilde{x}}\left(\tilde{h} \tilde{u}^{2}\right)+a \frac{\partial}{\partial \tilde{y}}(\tilde{h} \tilde{u} \tilde{v})=-a \frac{\partial}{\partial \tilde{x}}\left(\frac{\tilde{h}^{2}}{2}\right)-\mu \tilde{h} \frac{\tilde{u}}{\|\tilde{\mathbf{u}}\|}, \\
\sqrt{a} \frac{\partial}{\partial \tilde{t}}(\tilde{h} \tilde{v})+a \frac{\partial}{\partial \tilde{x}}(\tilde{h} \tilde{u} \tilde{v})+a \frac{\partial}{\partial \tilde{y}}\left(\tilde{h} \tilde{v}^{2}\right)=-a \frac{\partial}{\partial \tilde{y}}\left(\frac{\tilde{h}^{2}}{2}\right)-\mu \tilde{h} \frac{\tilde{v}}{\|\tilde{\mathbf{u}}\|} .
\end{gathered}
$$

The generic solution of the dimensionless system depends now only on $a$, not on the mass nor on acceleration due to gravity $g$. Such a result can be extended to more complex basal topography; however, this requires additional terms that can be shown again to only involve $a$ [see, e.g., Wieland et al., 1999; Savage and Hutter, 1989]. Beside the friction term, this invariance results from the fact that, for a leading order approximation, the vertical Lagrangian acceleration is assumed to be negligible leading to a hydrostatic state of pressure $p$. 
[20] Note that for models involving more complex friction behavior, such scaling must be investigated carefully. For example, the friction law proposed by Pouliquen [1999] for granular flows along a rough inclined rigid plane is expressed as

$$
\mu(\|\mathbf{u}\|, h)=\tan \delta_{1}+\left(\tan \delta_{2}-\tan \delta_{1}\right) \exp \left(-\beta \frac{h}{d} \frac{\sqrt{g h}}{\|\mathbf{u}\|}\right)
$$

where $\delta_{1}$ and $\delta_{2}$ are characteristic friction angles of the material, $d$ is a length scale, of the order of a grain diameter, and $\beta=0.136$ is a dimensionless parameter. With the scaling (7), such a law leads to

$$
\mu(\|\tilde{\mathbf{u}}\|, h)=\tan \delta_{1}+\left(\tan \delta_{2}-\tan \delta_{1}\right) \exp \left(-\beta \frac{H_{i}}{d} \frac{\tilde{h} \sqrt{\tilde{h}}}{\|\tilde{\mathbf{u}}\|}\right),
$$

and the $a$ scaling seems to be lost. However, $a$ scaling can be recovered by assuming that $d$ scales with either $H_{i}$ or $R_{i}$. Note that in the actual experimental conditions of Pouliquen [1999], $h / d \sim 10$ leading to $\beta h / d=O(1)$. The friction effect may therefore be sensitive to the ratio $H_{i} / d$, which in most granular experiments is simply the number of grains in the height of the initial granular mass. For large values of $H_{i}$, maintaining a constant $d$ value, the exponential becomes negligible and the friction angle is constant and quite small. In a LWA formulation, $d$ will appear as a new dissipation length scale that control the crossover between a constant and a variable friction coefficient. However, this remains quite speculative and the $d$ length must still be characterized for more general experimental conditions.

\subsection{Numerical Method}

[21] The numerical method used here to solve the hyperbolic system (1)-(3) relies on a finite volume formulation together with the hydrostatic reconstruction scheme developed by Audusse et al. [2004] for Saint Venant models, and on the well-balanced scheme of Bouchut [2004] to deal with friction. This finite volume scheme is second-order accurate, in contrast with the first-order method used by Mangeney-Castelnau et al. [2003] and based on a kinetic scheme. Alternative methods have recently been proposed by Denlinger and Iverson [2004], based on a combination of finite volume and finite element schemes, and by Pitman et al. [2003], based on a finite volume scheme together with an adaptive grid. A comparison of these different methods would be of interest but is clearly beyond the scope of this paper.

[22] We will now take a closer look at certain aspects of the method used. More details are given by Bouchut [2004]. For simplicity, we will consider only one dimension and the first order. The method has two parts: a method for solving the Saint Venant problem with topography and a procedure to interpret the source as an apparent topography.

\subsubsection{Saint Venant Problem With Topography}

[23] The one-dimensional Saint Venant system with topography is expressed by

$$
\begin{gathered}
\partial_{t} h+\partial_{x}(h u)=0 \\
\partial_{t}(h u)+\partial_{x}(h u 2+g h 2 / 2)+h Z_{x}=0,
\end{gathered}
$$

where $Z(x) / g$ represents topography. In this system the steady states given by $u=0, g h+Z=c s t$, play a crucial role. The numerical model is presented here with a term linked to the topography even though this term is equal to zero for the application studied here, i.e., the spreading of a granular column over a horizontal plane.

[24] In the past few years, a great deal of work has been devoted to the problem of finding well-balanced schemes for (13), i.e., schemes that preserve steady states at the discrete level. According to Bouchut [2004], such schemes can be written as

$$
U_{i}^{n+1}-U_{i}^{n}+\frac{\Delta t}{\Delta x}\left(F_{i+1 / 2-}-F_{i-1 / 2+}\right)=0,
$$

where $U_{i}^{n}$ is an approximation of $U=(h, h u)$ and the left/ right numerical fluxes are computed as

$$
\begin{gathered}
F_{i+1 / 2-}=F_{l}\left(U_{i}, U_{i+1}, \Delta Z_{i+1 / 2}\right) \\
F_{i+1 / 2+}=F_{r}\left(U_{i}, U_{i+1}, \Delta Z_{i+1 / 2}\right),
\end{gathered}
$$

with $\Delta Z_{i+1 / 2}=Z_{i+1}-Z_{i}$. The numerical fluxes $F_{l}$ and $F_{r}$ must satisfy two consistency properties. The first is consistency with the conservative term,

$$
F_{l}(U, U, 0)=F_{r}(U, U, 0)=F(U) \equiv(h u, h u 2+g h 2 / 2),
$$

and the second is the consistency with the source,

$$
F_{r}\left(U_{l}, U_{r}, \Delta Z\right)-F_{l}\left(U_{l}, U_{r}, \Delta Z\right)=(0,-h \Delta Z)+o(\Delta Z),
$$

as $U_{l}, U_{r} \rightarrow U$ and $\Delta Z \rightarrow 0$. An overall property that must be satisfied is the conservation of mass,

$$
F_{l}^{h}\left(U_{l}, U_{r}, \Delta Z\right)=F_{r}^{h}\left(U_{l}, U_{r}, \Delta Z\right) \equiv F^{h}\left(U_{l}, U_{r}, \Delta Z\right)
$$

A well-balanced scheme must satisfy

$$
F_{i+1 / 2-}=F\left(U_{i}\right) \quad F_{i+1 / 2+}=F\left(U_{i+1}\right)
$$

whenever

$$
u_{i}=u_{i+1}=0 \quad g h_{i+1}-g h_{i}+\Delta Z_{i+1 / 2}=0 .
$$

A consistent well-balanced scheme capable of dealing with transonic flows and dry states $h=0$, that satisfies a discrete entropy inequality and is very inexpensive has been proposed recently by Audusse et al. [2004]. It is called the hydrostatic reconstruction method, and has the following form:

$$
F_{l}\left(U_{l}, U_{r}, \Delta Z\right)=\mathcal{F}\left(U_{l}^{*}, U_{r}^{*}\right)+\left(\frac{g}{2} h_{l} 2-\frac{g}{2} h_{l_{*}}^{2}\right)
$$

$$
F_{r}\left(U_{l}, U_{r}, \Delta Z\right)=\mathcal{F}\left(U_{l}^{*}, U_{r}^{*}\right)+\left(\frac{g}{2} h_{r}{ }^{0}-\frac{g}{2} h_{r^{*}}^{2}\right),
$$


where $U^{*}=\left(h_{l *}, h_{l *} u_{l}\right), U_{r}^{*}=\left(h_{r^{*}}, h_{r^{*}} u_{r}\right)$,

$$
\begin{gathered}
h_{l_{*}}=\max \left(0, h_{l}-\max (0, \Delta Z / g)\right), \\
h_{r^{*}}=\max \left(0, h_{r}-\max (0,-\Delta Z / g)\right) .
\end{gathered}
$$

Here $\mathcal{F}$ is any entropy providing a consistent numerical flux for the homogeneous problem (i.e., with $Z=c s t$ ), that is capable of dealing with dry states. We use a relaxation solver described by Bouchut [2004], but other choices give similar results.

\subsubsection{Friction as an Apparent Topography}

[25] The apparent topography method is general and can be used to deal with generic source terms [see Bouchut, 2004]. Note that the apparent topography introduced here is only a numerical ruse used to solve the equations and has nothing to do with any physical assumption concerning the rheological behavior. For our purpose, consider the Saint Venant system with friction

$$
\begin{gathered}
\partial_{t} h+\partial_{x}(h u)=0 \\
\partial_{t}(h u)+\partial_{x}(h u 2+g h 2 / 2)+h Z_{x}=h f,
\end{gathered}
$$

where $Z=Z(x)$, and $f=f(t, x)$ must satisfy

$$
\begin{aligned}
& |f(t, x)| \leq g \mu \\
& u(t, x) \neq 0 \Rightarrow f(t, x)=-g \mu \frac{u(t, x)}{|u(t, x)|} .
\end{aligned}
$$

Solutions at rest are given by $u=0, f=\partial_{x}(g h+Z)$ or, equivalently,

$$
u=0 \quad \text { and } \quad\left|\partial_{x}(g h+Z)\right| \leq g \mu
$$

The idea is to identify the equations in (22) as (13) with a new topography $Z+B$, where $\partial_{x} B=-f$. $B$ also depends on time while it should be time-independent; thus we take $\partial_{x} B^{n}=-f^{n}$ and solve (13) over the time interval $\left(t_{n}, t_{n+1}\right)$ with topography $Z+B^{n}$. In this way, we freeze the source term on a time interval. The estimation of the source term is of course exact for a stationary solution.

[26] At the discrete level, this is done as follows. We define

$$
\Delta B_{i+1 / 2}^{n}=-f_{i+1 / 2}^{n} \Delta x
$$

and update $U=(h, h u)$ via

$$
U_{i}^{n+1}-U_{i}^{n}+\frac{\Delta t}{\Delta x}\left(F_{i+1 / 2-}-F_{i-1 / 2+}\right)=0,
$$

with

$$
\begin{gathered}
F_{i+1 / 2-}=F_{l}\left(U_{i}, U_{i+1}, \Delta Z_{i+1 / 2}+\Delta B_{i+1 / 2}^{n}\right) \\
F_{i+1 / 2+}=F_{r}\left(U_{i}, U_{i+1}, \Delta Z_{i+1 / 2}+\Delta B_{i+1 / 2}^{n}\right),
\end{gathered}
$$

where the numerical fluxes $F_{l}, F_{r}$ are those associated with the problem of section 3.3.1 without a source. Then if $f_{i+1 / 2}^{n}$ is a consistent value for the friction, it is easy to see that our scheme is consistent with (22) and well-balanced since it preserves the discrete steady states satisfying $u_{i}=$ $u_{i+1}=0$ and $g h_{i+1}-g h_{i}+\Delta Z_{i+1 / 2}=f_{i+1 / 2} \Delta x$. The scheme also conserves mass, is capable of computing dry bed states and satisfies a discrete entropy inequality. For the computation of $f_{i+1 / 2}^{n}$, a good choice is

$$
f_{i+1 / 2}^{n}=-\underset{g \mu}{\operatorname{proj}}\left(\frac{g h_{i}-g h_{i+1}-\Delta Z_{i+1 / 2}}{\Delta x}+\frac{u_{i+1 / 2}}{\Delta t}\right),
$$

where

$$
\underset{g \mu}{\operatorname{proj}}(X)= \begin{cases}X & |X| \leq g \mu, \\ g \mu \frac{X}{|X|} & |X|>g \mu,\end{cases}
$$

and, for example,

$$
u_{i+1 / 2}=\frac{h_{i} u_{i}+h_{i+1} u_{i+1}}{h_{i}+h_{i+1}} .
$$

This gives a well-balanced scheme, in the sense that data satisfying

$$
u_{i}=0 \quad \text { and } \quad\left|g h_{i}-g h_{i+1}-\Delta Z_{i+1 / 2}\right| \leq g \mu \Delta x
$$

are preserved exactly.

\subsubsection{Two-Dimensional Scheme}

[27] The method can be extended to two dimensions in a relatively direct manner (by resolving interface problems) that will not be described here. For our purpose, we performed a series of numerical experiments on a twodimensional regular grid with $400 \times 400$ points, for different values of the aspect ratio $a$ and initial radius $R_{i}$.

\section{Granular Mass Changes With Time}

[28] We first investigate the ability of our minimal model to capture the different regimes observed experimentally for the spreading of a granular mass and to describe the resulting morphology of the deposit.

[29] In the numerical experiments, initial conditions are defined in terms of an initial cylindrical mass of height $H_{i}$ and of radius $R_{i}$ with zero initial velocities. A lower cutoff, $\epsilon=7 \times 10^{-4} \mathrm{~m}$, is introduced numerically when computing the $h$ profiles in order to simulate the lower cutoff inherent to the experimental resolution of the $h$ measures, estimated by Lajeunesse et al. [2004] to be of the order of two to three grain diameters.

[30] On the basis of the three types of deposits observed experimentally, three numerical experiments have been performed corresponding to the following initial geometries: (1) $a=0.56, R_{i}=70.5 \mathrm{~mm}$, (2) $a=0.8, R_{i}=70.5 \mathrm{~mm}$, and (3) $a=5.4, R_{i}=28 \mathrm{~mm}$. The cases 1 and 2 correspond to the first dynamic regime and case 3 to the second regime described by Lajeunesse et al. [2004].

\subsection{Calibration of the Model}

[31] The effective friction law (4) in our minimal model is characterized by a single parameter, i.e., the limiting angle of stability $\delta$. This angle could be directly measured by 

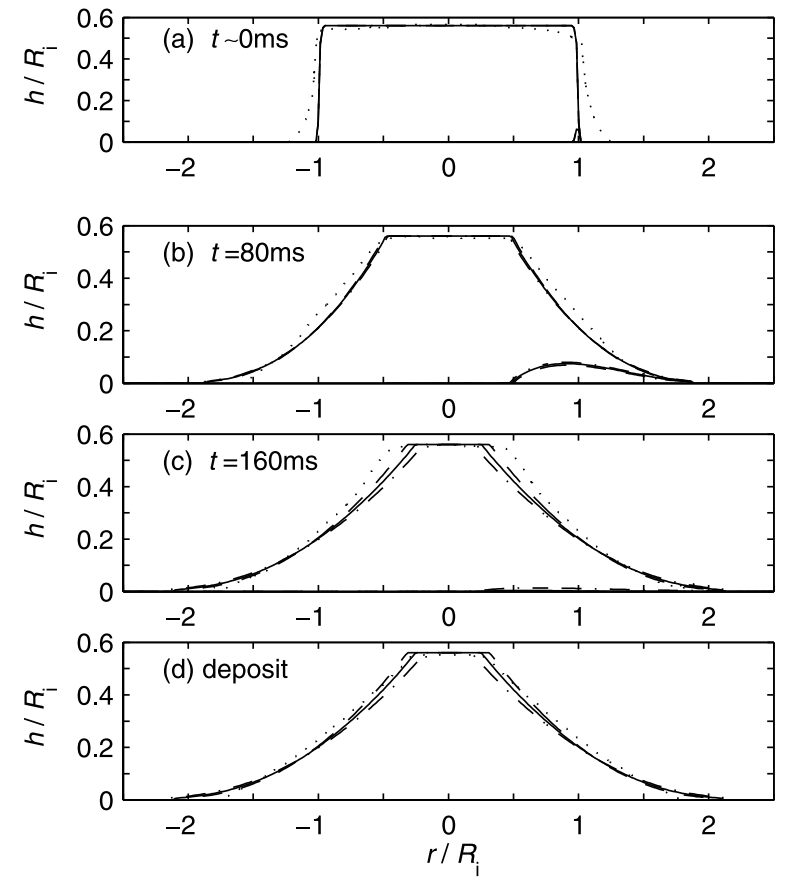

Figure 2. Evolution of the profile $h\left(r / R_{i}, t\right) / R_{i}$ and corresponding fluxes $h u\left(r / R_{i}, t\right) /\left(R_{i} \sqrt{g H_{i}}\right)$ during the spreading of a granular mass, for three different friction angles: $\delta=30^{\circ}$ (dash-dotted lines), $\delta=32^{\circ}$ (solid lines), and $\delta=34^{\circ}$ (dashed lines). The initial geometry of the granular mass is $a=0.56$, and $R_{i}=70.5 \mathrm{~mm}$. Results for the laboratory experiment are also plotted here as dotted lines.

performing release experiments on an initial conical mass. Unfortunately such experiments were not performed by Lajeunesse et al. [2004] and results were not available at the time of this study. Therefore a simple procedure was adopted: the friction angle $\delta$ was first estimated by direct comparison between laboratory experiments involving the most favorable case, case 1, for which precise experimental observations were available, and then kept constant for the other cases.

[32] Numerical simulations show very similar dynamics for different values of the friction angle $\delta, \delta \in\left[32^{\circ}, 34^{\circ}\right]$, until roughly $t \simeq 120 \mathrm{~ms}$ (Figure 2). The height $h$ and flux $h u$ profiles, corresponding to $\delta=30^{\circ}, \delta=32^{\circ}$, and $\delta=34^{\circ}$, are found to be almost identical as shown in Figure 2b for $t=$ $80 \mathrm{~ms}$. Even though the deposit appears to depend weakly on the friction angle, differences in the $h$ and $h u$ profiles appear just before and during the arrest phase, as experimentally observed within the range of friction angles $\left(30^{\circ}<\right.$ $\delta<35^{\circ}$ ) explored by Lube et al. [2004] using different types of material such as sand, salt, couscous grains, rice or sugar.

[33] Numerically, mass spreading is found to stop at $t \sim$ $160 \mathrm{~ms}$, for $\delta=34^{\circ}$. The final deposit is obtained at $t \sim$ $180 \mathrm{~ms}$ for all the three values of $\delta$ as shown in Figures 2c and $2 \mathrm{~d}$. The main differences between the three profiles are observed at the top while the front region remains quite similar. The ratio $R_{f} / R_{i}$ is found to be a relatively robust parameter as already observed for spreading experiments with glass beads, for various diameters and for both rigid and erodible planar surfaces [Lajeunesse et al., 2004].
[34] The best agreement between laboratory and numerical observations is obtained for a friction angle $\delta=32^{\circ}$. This value was selected and used for all the numerical experiments presented below.

\subsection{Comparison Between Numerical and Experimental Results}

[35] For small aspect ratios, $a<1$, the overall collapse and spreading observed in laboratory is globally represented numerically by the model. The dam-break-type behavior of the profile of the granular mass with time, measured by laboratory experiments, is well reproduced by the numerical experiments, as observed in Figure 3 for the initial aspect ratios $a=0.56$ and $a=0.8$. Moreover, the behaviors of the front and the deposit are found to be in very good agreement with the laboratory measurements, and differences only appear in the vicinity of the highest zone of the deposit and at the front. The deposit in the numerical experiment, corresponding to $a=0.8$, exhibits a slightly steeper profile in the highest region than actually measured in the laboratory experiment. The front, for both $a=0.56$ and $a=0.8$, is found to be more spread out in numerical experiments than in laboratory experiments. While the position of the front, measured in laboratory and numerical experiments, progresses in a very similar way during most of the experiment (see Figure $3 \mathrm{c}$ for $a=0.56$ at $t=160 \mathrm{~ms}$ and Figure $3 \mathrm{~g}$ for $a=0.8$ at $t=180 \mathrm{~ms}$ ), the final movement of the front is found in laboratory experiments to progressively retropropagate (see Figures $3 d$ and $3 \mathrm{~h}$ ). Such retropropagation may be explained by a loss of mass at the front during the arrest phase in laboratory experiments.

[36] Numerical estimations of the times at which the front stops are of the same order as those measured in the laboratory by Lube et al. [2004], although slightly lower. For $a=0.56$, Lube et al. [2004] actually measured a normalized time $t / \sqrt{R_{i} / g} \sim 2.2$ leading to $t \sim 186 \mathrm{~ms}$, while numerical estimations give $t_{s} \sim 170 \mathrm{~ms}$. For $a=0.8$, laboratory estimations are $t / \sqrt{R_{i} / g} \sim 2.7$ leading to $t \sim$ $230 \mathrm{~ms}$, while numerical estimations give $t_{s} \sim 200 \mathrm{~ms}$. However, when taking a closer look, the arrest phase appears quite different in the numerical experiments compared to the actual observations of Lajeunesse et al. [2004] for laboratory experiments. In laboratory experiments, for both $a=0.56$ and $a=0.8$, the arrest phase is observed to start at the front edge of the spreading mass and then to propagate toward the highest zone. When the front stops, a late relaxation of the highest deposit region is observed leading to a smaller slope after $t=180 \mathrm{~ms}$ for $a=0.56$ (see Figures $3 \mathrm{c}$ and $3 \mathrm{~d}$ ) and after $t=200 \mathrm{~ms}$ for $a=0.8$ (see Figures $3 \mathrm{~g}$ and $3 \mathrm{~h}$ ). This relaxation seems to be accommodated by shallow surface flows smoothing out the highest zone of the deposit. In numerical experiments, the arrest phase is different for $a=0.56$ and $a=0.8$. For $a=0.56$, it appears to initiate in the vicinity of the front (see Figure 4a at $t=162 \mathrm{~ms}$ ) and then to propagate both toward the front edge and the center of the mass. Finally, the center of the mass first comes to rest at $t=167 \mathrm{~ms}$ (see Figures $4 \mathrm{~b}$ and $4 \mathrm{c}$ ), while the whole mass comes to rest only later. For $a=$ 0.8 , the arrest phase appears to start in the central part of the granular mass and then to propagate outward from the center to the spreading front (see Figures $4 d-4 f$ ). In laboratory experiments, arrest phase propagation is found 

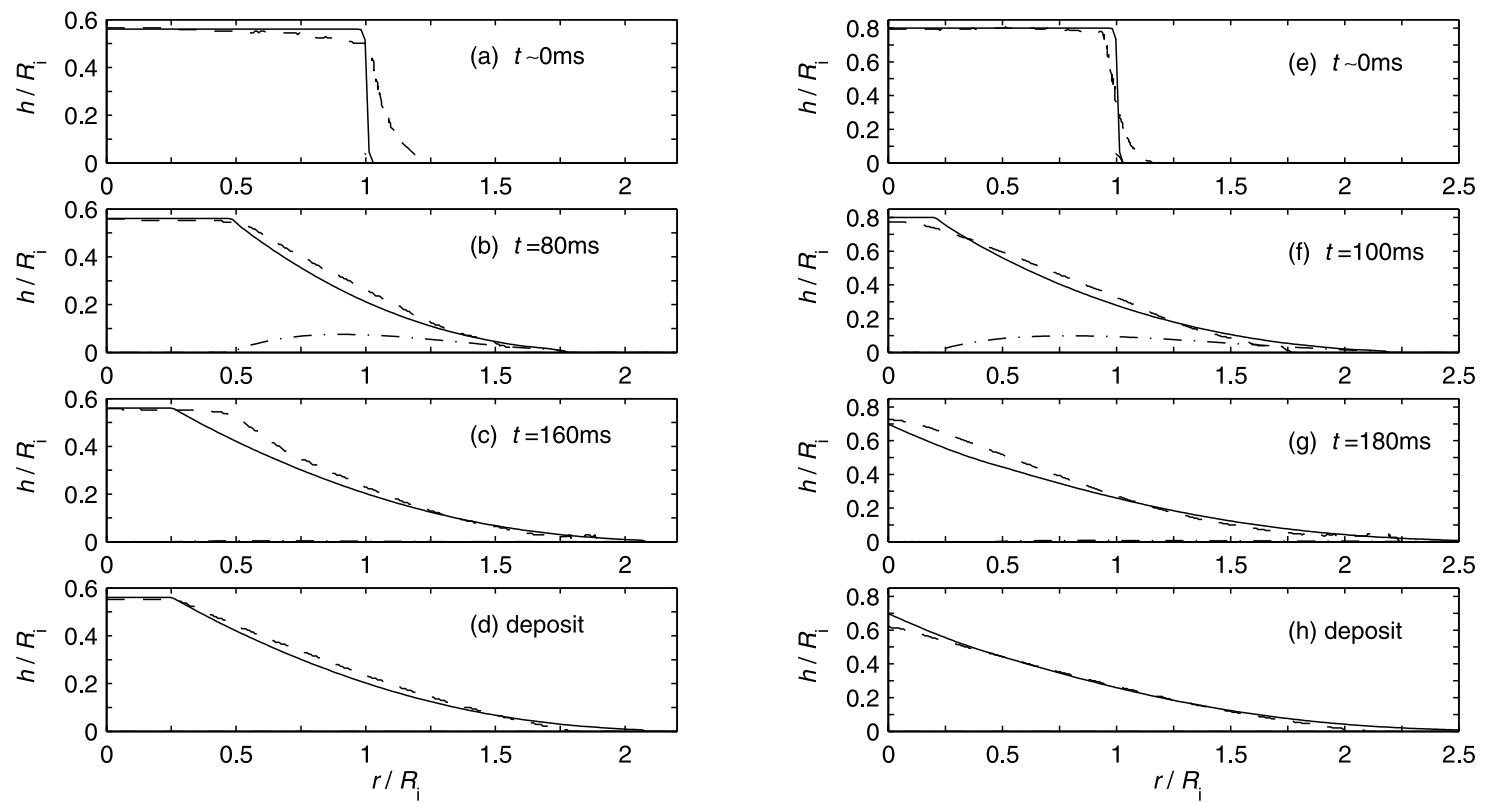

Figure 3. Evolution of the profiles $h\left(r / R_{i}, t\right) / R_{i}$ (solid lines) and fluxes $h u\left(r / R_{i}, t\right) /\left(R_{i} \sqrt{g H_{i}}\right)$ (dash-dotted lines) during the spreading of a granular mass with a friction angle $\delta=32^{\circ}$. (a-d) Experiment 1: $a=0.56$, $R_{i}=70.5 \mathrm{~mm}$. (e-h) Experiment 2: $a=0.8, R_{i}=70.5 \mathrm{~mm}$. Experimental data are plotted for reference using dashed lines.

to last much longer than in numerical experiments. Note that experimentally, the arrest phase is actually observed at the top surface of the granular mass by Lajeunesse et al. [2004] and Lube et al. [2004] have suggested that differences may be expected when the arrest phase is observed at the base of the mass.

[37] For high aspect ratios, $a>1$, the granular mass is found (see Figure 5 for $a=5.4$ ) to spread much faster in numerical experiments than in laboratory experiments, where the initial cylindrical shape is also preserved during the spreading. However, even if the dynamics of the granular mass are not reproduced by the numerical experi- ments in these cases, the final deposit is relatively well represented (see Figure 5d for $a=5.4$ ). This may suggest the final deposit to be the result of a global energy balance. Dissipation related to the inertial vertical motion is expected to play an important role as the initial aspect ratio increases, and to extend outside the domain of validity of the LWA. On the other hand, part of the mass mobility observed in numerical experiments could also suggest that the minimal model must be extended by incorporating a more sophisticated constitutive behavior as discussed later in this paper.

[38] Numerical experiments suggest that the first step of the spreading, involving fast inertial motion during which
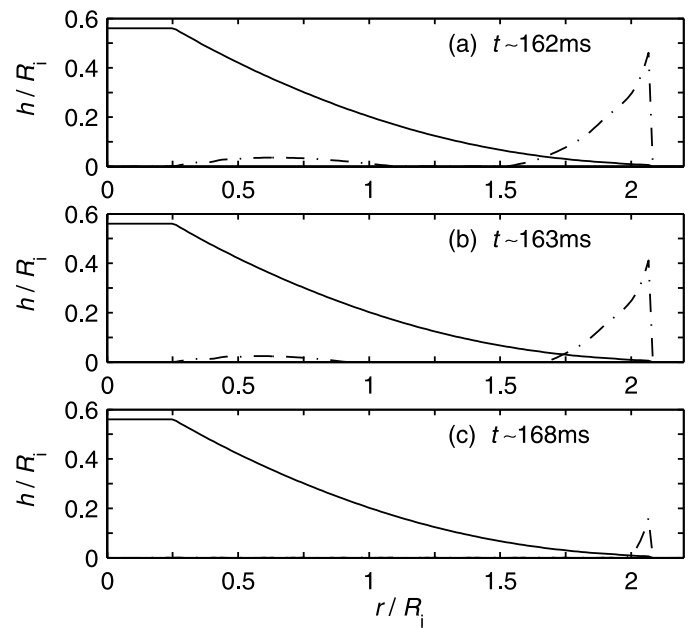
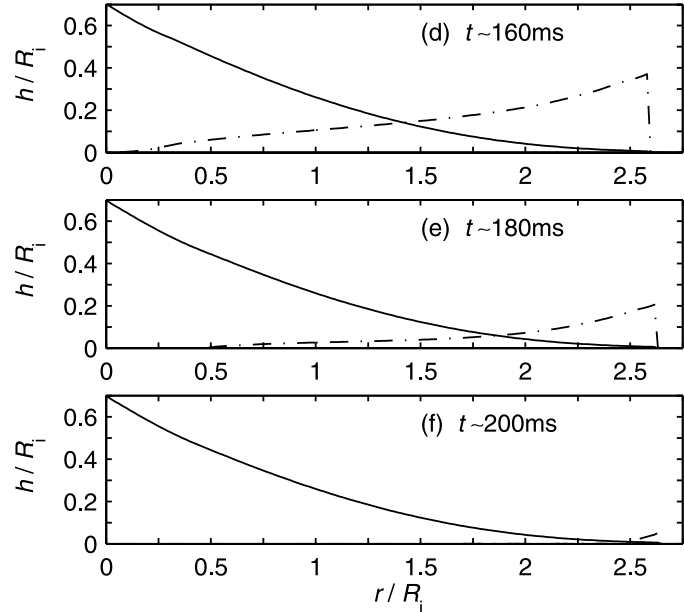

Figure 4. Evolution of the $h(r, t) / R_{i}$ profile (solid lines) and corresponding velocity $u(r, t) / \sqrt{g R_{i}}$ (dashdotted lines) as a function of the normalized radius $r / R_{i}$ during the arrest phase (a-c) for $a=0.56, R_{i}=$ $70.5 \mathrm{~mm}$ where $5 u(r, t) / \sqrt{g R_{i}}$ has been represented for better visibility and $(\mathrm{d}-\mathrm{f})$ for $a=0.8, R_{i}=$ $70.5 \mathrm{~mm}$. 

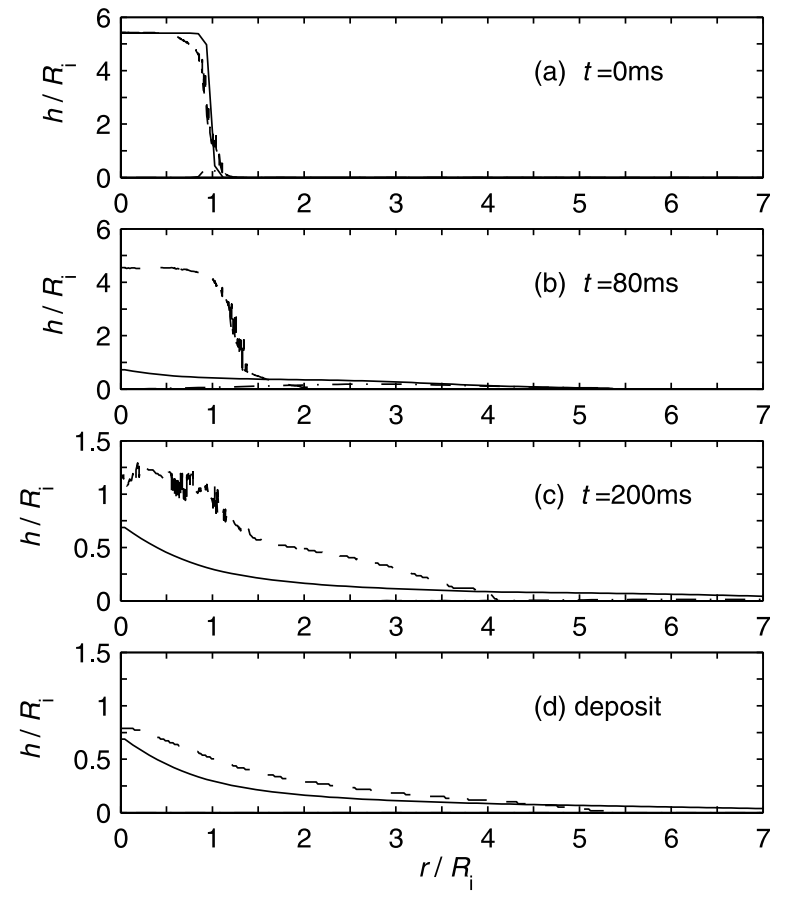

Figure 5. Evolution of the $h\left(r / R_{i}, t\right) / R_{i}$ profile (solid lines) and corresponding fluxes $h u\left(r / R_{i}, t\right) /\left(R_{i} \sqrt{g R_{i}}\right)$ (dash-dotted lines) of the spreading of a granular mass for $\delta=32^{\circ}$ and experiment 3: $a=5.4, R_{i}=28 \mathrm{~mm}$. Experimental data are plotted using dashed lines.

most of the final morphology of the deposit is created, is correctly captured by the model for low inertial aspect ratios. In that regime, the inertial motion is expected to involve the granular mass without significant vertical fluctuations and dissipation. The late relaxation observed in laboratory experiments when the front has stopped, seems to be accommodated by very shallow surface avalanches involving a few glass beads as the result of a vertical propagation of a fluid to static interface. Such a consolidation phase may be related either to some steric aspects or a basal pore pressure decrease. Detection of this phase by the model would require an extension of the LWA or a more sophisticated representation of the constitutive behavior.

\subsection{Influence of the Initial Conditions}

[39] Identification of appropriate initial conditions is always difficult when performing numerical experiments, especially in the case of a high initial aspect ratio. This is directly related to the uncontrolled conditions of the laboratory experiments where the granular mass has begun to spread in the time required to remove the cylinder (e.g., see dotted lines in Figure 2a).

[40] Instead of imposing an initial perfectly cylindrical shape in the numerical experiments, two different initial profiles have been considered, based on the actual laboratory experiments: (1) a profile as measured experimentally just after removing the cylinder as shown by the dashed lines in Figure 6a and (2) a profile as measured experimentally at $t=20 \mathrm{~ms}$ and exhibiting an edge at $r / R_{i} \sim 1$, as shown by the solid lines in Figure 6b. In order to maintain symmetric initial conditions, the laboratory profiles are here symmetrized by imposing the left part of the experimental profile $(h(r) ; r \leq 0)$ for $r \leq 0$ and $r \geq 0$ (Figures 6a and 6b). Note that even if these laboratory-derived profiles are actually associated with a given velocity field, a zero initial velocity has been imposed numerically. The full laboratoryderived profiles, $r \geq 0$ and $r \leq 0$, are shown in Figure 6 to show their initial asymmetry. This initial asymmetry of the laboratory-derived profiles is in fact progressively smoothed out during experiments (Figure 6) even though the use of initial conditions reconstructed from the left part of the laboratory profiles led to a slightly better agreement between numerical and laboratory experiments (Figure 6). Interestingly enough, it is found numerically that the mass stops approximately at the same time whatever the initial conditions (Figure 6e). When compared with perfectly cylindrical initial conditions (Figure 3c), a better agreement between numerical and laboratory experiments is observed for these new initial conditions at time $t=160 \mathrm{~ms}$ and the

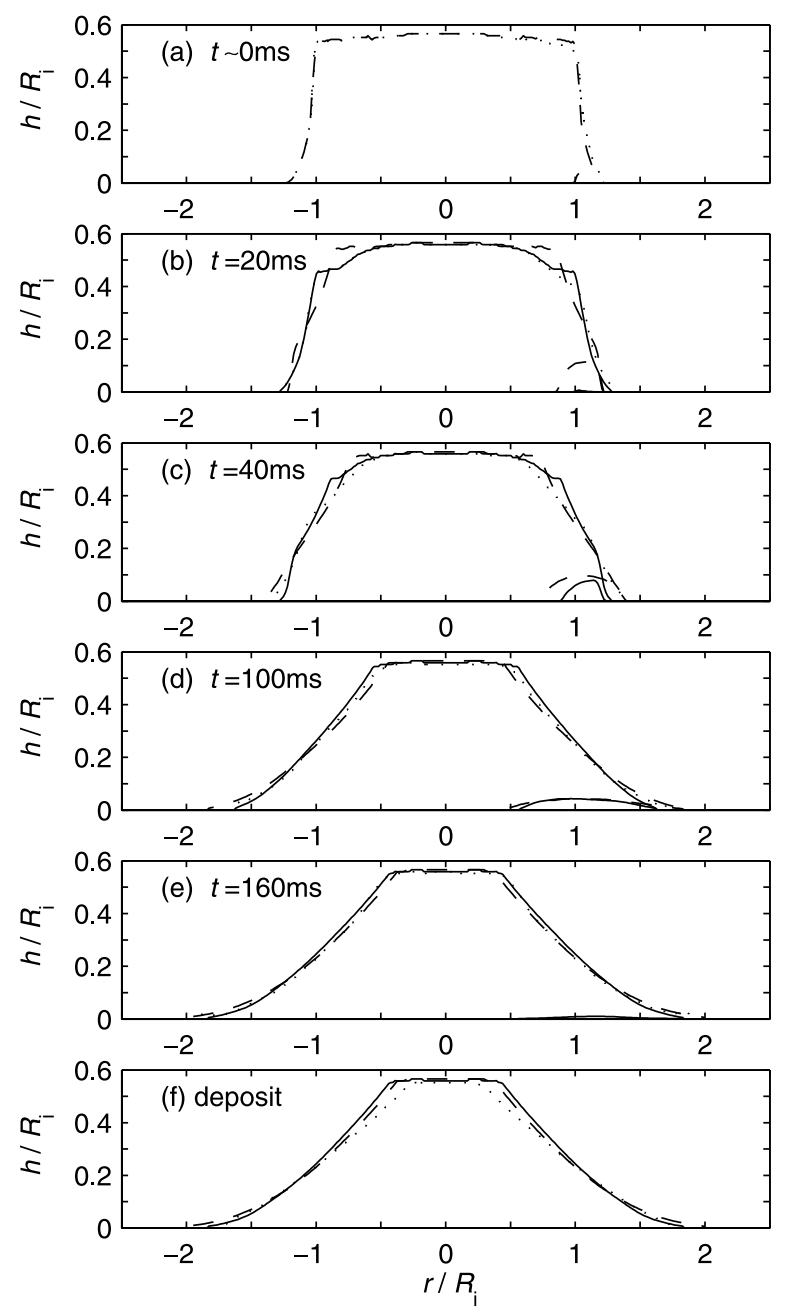

Figure 6. Evolution of the $h(r, t) / R_{i}$ profile and corresponding fluxes $h u(r, t) /\left(R_{i} \sqrt{g R_{i}}\right)$ of the spreading of a granular mass with $a=0.56, R_{i}=70.5 \mathrm{~mm}$ using $\delta=32^{\circ}$ as a function of $r / R_{i}$ for initial conditions corresponding to the symmetrized experimental profile obtained just after the lifting of the tube (dashed lines), and $20 \mathrm{~ms}$ after the lifting of the tube (solid lines). Experimental data are plotted with dotted lines. 
spreading is very accurately reproduced until the end of the spreading at time $t \sim 170 \mathrm{~ms}$.

[41] For high initial aspect ratio, as previously mentioned, an edge located at $r / R_{i} \sim 1$ is observed in the laboratory experiments, probably related to nonhomogeneous initial loading conditions, and is progressively smoothed out during the experiment. Such an edge does not appear in the profiles derived from numerical experiments based on an initial cylindrical profile or the laboratory-derived profile 1. The exact mechanism that leads to such an edge pattern, which has also been observed by Daerr and Douady [1999] in a slightly different context, remains open to question. When the laboratory-derived profile 2 is used initially, the edge pattern is actually initially imposed in the numerical experiment. However, even in this case, the edge pattern does not evolve as observed in laboratory experiments. While the edge pattern remains stationary and is progressively smoothed out in laboratory experiments (Figures $6 \mathrm{~b}$ and $6 \mathrm{c}$ ), the edge propagates in numerical experiments, as a shock wave, toward the highest region without smoothing. A discrete element simulation by Müller [1995] of granular collapse experiments, somewhat related to this study, does seem to reproduce the laboratory observed type of evolution of such an edge pattern, which can be related in this study to the localization of the deformation within the granular mass and the formation of a slip surface. Such a step pattern would therefore be related to nonhomogeneous deformation and shear zone evolution within the granular mass, a mode of deformation that is clearly outside the conditions for validity of the LWA and any vertically integrated model. On the basis of the numerical experiments, the occurrence of such an edge pattern does not seem to alter the global averaged spreading and the final deposit that are mostly controlled by the overall energy imbalance associated with the initial collapse conditions, rather than by second-order nonhomogeneity effects, consistent with the underlying principles of depth-average models.

\subsection{Limits of Saint Venant Equations}

[42] Despite the kinematic assumptions and the vertical integration of the governing equations of the minimal model, laboratory experiments of the spreading of granular mass with small initial aspect ratios are well reproduced by numerical experiments until the arrest of the spreading front. For these configurations, we would expect the main shape changes of the granular mass, and in particular the final deposit, to be correctly depicted by the macroscopic balance inferred by the integrated governing equations of the model despite some possible localization of the deformation within the bulk.

[43] This comparison confirms the existence of two regimes. The first, for low initial aspect ratios, corresponds to experiments $a=0.56$ and $a=0.8$ for which the model provides an appropriate description, and the second, for high initial aspect ratios, corresponds to experiment $a=5.4$ for which the dynamics are not correctly modeled. This interpretation is supported by the observed laboratory scaling of the front velocity $V$. For $a<3$ (regime 1), $V$ scales with the celerity of gravity waves for shallow flow $\sqrt{g H_{i}}$, while for $a>3$ (regime 2), it scales with $\sqrt{g R_{i}}$ [Lajeunesse et al., 2004]. In the first regime, spreading occurs as a result of surface avalanches, for which vertical acceleration remains negligible compared to the vertical gradient of the pressure. In the second regime, spreading results from a vertical collapse. In that case, vertical accelerations and vertical velocities may become significant and incompatible with the physical assumptions of the depth-integrated model. Moreover, such a dynamic initial vertical collapse may generate high air pressures in the pores of the granular material, and pore pressure changes within the granular mass may play a paramount role in the propagation of spreading during which an undrained behavior could prevail. Note once again that the existence of a small flow aspect ratio, a condition generally used for scaling arguments is a sufficient but not necessary condition for the LWA which relies on kinematic scaling arguments between the vertical and horizontal velocity fields and on the vertical acceleration field, as illustrated in various fields of geophysics such as ice sheet flow modeling, where the shallow ice approximation has been shown to accurately reproduce the flow of the ice in the dome region [Mangeney and Califano, 1998]. Part of the LWA ability to depict these experiments is also expected to be related to the fact that the initial geometry is rapidly smoothed out, leading to lower flow aspect ratio geometries during the dynamic phase. Furthermore, the region located at a distance $r$ from the center of the mass $r>R_{i}$ is subject to shallow flow while the region $r<R_{i}$ is expected to involve essentially surface flow. Although a model involving both static and fluid granular layers [Douady et al., 1999; Aranson and Tsimring, 2002] would probably provide a more appropriate framework to describe the flow for $r<R_{i}$, the simple LWA is still expected to represent the dynamics when a significant part of the column is actually flowing and when the static fluid interface does not vary vertically to a great extent in space and time.

[44] When the mass begins to stop, an arrest phase is observed both in laboratory and numerical experiments, and propagates within the granular mass. The disagreement between laboratory and numerical observations of this arrest phase suggest that our minimal model should be extended to capture a more complex behavior of the granular mass during spreading. Note also that laboratory observations show differences in the arrest phase when measured from the top or the base of the granular mass [Lube et al., 2004]. Laboratory experiments suggest that the arrest phase involves both a horizontal and a vertical propagation of transition between static and fluid material, with a vertical propagation toward the surface inducing shallower avalanches. Such a consolidation phase cannot be reproduced by the minimal model in its present formulation. Such a static/fluid boundary propagation may be explicitly taken into account within the framework developed by Douady et al. [1999] or Aranson and Tsimring [2002]. This consolidation phase could also be related to a pore pressure decrease at the base of the granular material and would require, in this case, a kind of mixture theory taking into account effective stresses and interactions between a solid skeleton and pore fluids [Pastor et al., 2002; Iverson and Denlinger, 2001].

[45] The incapacity to correctly reproduce the occurrence and the changes of the edge pattern observed in laboratory experiments, as previously discussed, may be related to the fact that vertically integrated models cannot correctly de- 

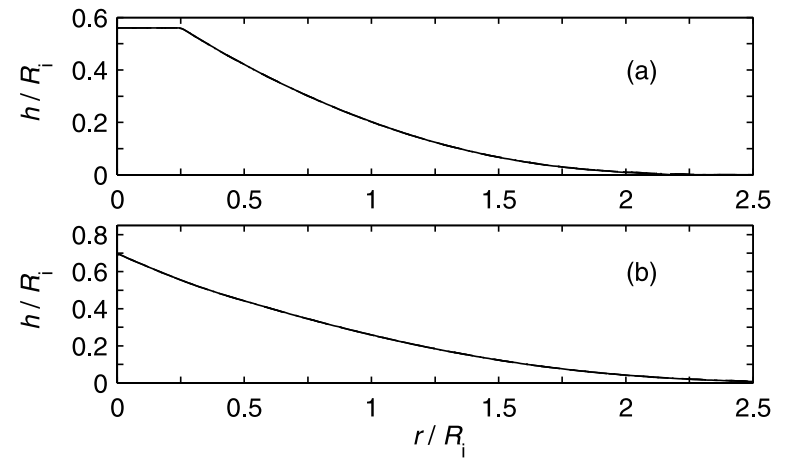

Figure 7. Profiles of the normalized deposit $h / R_{i}$ as a function of $r / R_{i}$ calculated with $R_{i}=70.5 \mathrm{~mm}, R_{i} / 2,2 R_{i}$, and $10^{4} R_{i}$ for (a) $a=0.56$ and (b) $a=0.8$.

scribed vertically nonhomogeneous deformation modes. Moreover when introduced initially, the edge evolution observed in the numerical experiments is reminiscent of that of a shock wave with zero thickness. Introduction of both a static and flowing phase, or of a mixture theory, would lead to parabolic-hyperbolic equations, allowing shocks with nonzero thickness and different propagation/ diffusion modes.

[46] Detailed analysis of the heterogeneous behavior of a collapsing granular mass requires the use of numerical models at small scale using discrete elements [e.g., Müller, 1995; Staron et al., 2002]. Although this is beyond the scope of this study, we will note here some of the results of this type of simulation. A discrete element simulation of the collapse of a rectangular granular mass was performed by Müller [1995]. This numerical experiment is quite different since the beads flow over a smooth bed and the granular mass reaches the right end of the box before the front stops. Moreover, the initial ratio $H_{i} / d$, where $d$ is a characteristic bead diameter, is of the order of 20 whereas $H_{i} / d \sim 100$ in the spreading experiments of Lajeunesse et al. [2004] and Lube et al. [2004]. Nevertheless, these simulations provide some insight into the flow dynamics. First a relatively deep avalanche occurs along an inclined surface of rupture of about $60^{\circ}$, then the motion propagates within the granular mass leading to a relatively stable avalanche along the surface of slope $30^{\circ}$ with a shallow horizontal flow in the front part. The existence of a first and second fracture was also experimentally observed by Daerr and Douady [1999] and is consistent with the interpretation made by Lajeunesse et al. [2004]. The minimal model considered here, based on a vertically integrated model, cannot describe such mechanisms. However, as suggested in section 4.3, the details of the initial collapse seem to have a negligible effect on the mean behavior of the flow and on the final deposit. The comparison between continuum and discrete modeling requires further investigation, which is in progress.

\section{Aspect Ratio $a$, a Key Parameter}

\subsection{A Mass- and Gravity-Independent Solution}

[47] In section 3.2, the governing equations of the minimal model are shown to satisfy the scale invariance observed in the laboratory. This has been investigated numerically, and normalized profiles of the final deposit are shown in Figure 7 for four numerical experiments corresponding to the same initial aspect ratios $a=0.56$ (Figure 7a) and $a=0.8$ (Figure 7b) but to different masses with initial radii $R_{i} / 2,2 R_{i}, 10^{4} R_{i}$, and $R_{i}=70.5 \mathrm{~mm}$. The values of $H_{i}$ are obviously scaled by the same factor to keep a constant aspect ratio $a$. The initial volume of the cylinder being $V_{i}=\Pi R_{i}^{2} H_{i}$, these experiments involve masses that are 8 times smaller, 8 times larger, and $10^{12}$ times larger, respectively, with a mass as high as $M \sim 10^{9} \mathrm{~m}^{3}$. The four normalized profiles match perfectly, indicating that the numerical model is accurate. The same result is obtained for $a=5.4$.

\subsection{Characteristics of the Deposit Morphology}

[48] Although the relevance of the numerical experiments for initial aspect ratios $a>1$ remains questionable due to the high vertical accelerations, the characteristics of the associated deposit turn out to be somewhat similar to those obtained in the laboratory and will be discussed together with the results obtained for small initial aspect ratios.

[49] As described in section 2, three distinct deposit morphologies have been observed in laboratory experiments, depending on the initial aspect ratio $a$ of the released granular mass [Lajeunesse et al., 2004]. Two of these morphologies, associated with the small initial aspect ratio spreading regime, are well reproduced by numerical experiments, i.e., the "truncated morphology" (Figure 3d for $a=$ 0.56 ) and the conical morphology (Figure $3 \mathrm{~h}$ for $a=0.8$ ). The laboratory transition at $a \sim 0.7$ between the two morphologies is very well represented by the numerical experiments, as shown in Figure 8. The slight difference in the predicted values of the transition, $a=0.74$ for numerical experiments and $a=0.74$ for laboratory experiments, is within the uncertainty level of the experiments.

[50] Another transition was observed in the laboratory around $a=3$. For $a>3$, the height profiles are characterized by an inflection point. This inflection point is also observed
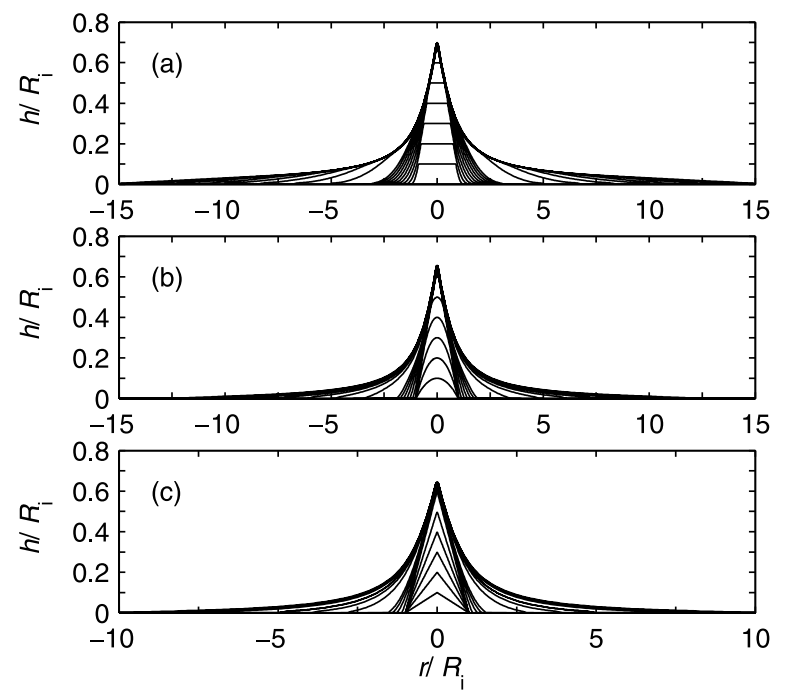

Figure 8. Normalized profiles of the deposit as a function of the normalized radius for initial (a) cylindrical (b) paraboloidal, and (c) conical mass. The parameter $a$ varies from 0.1 to 1 in intervals of 0.1 and from 1 to 10 in intervals of 1 . 

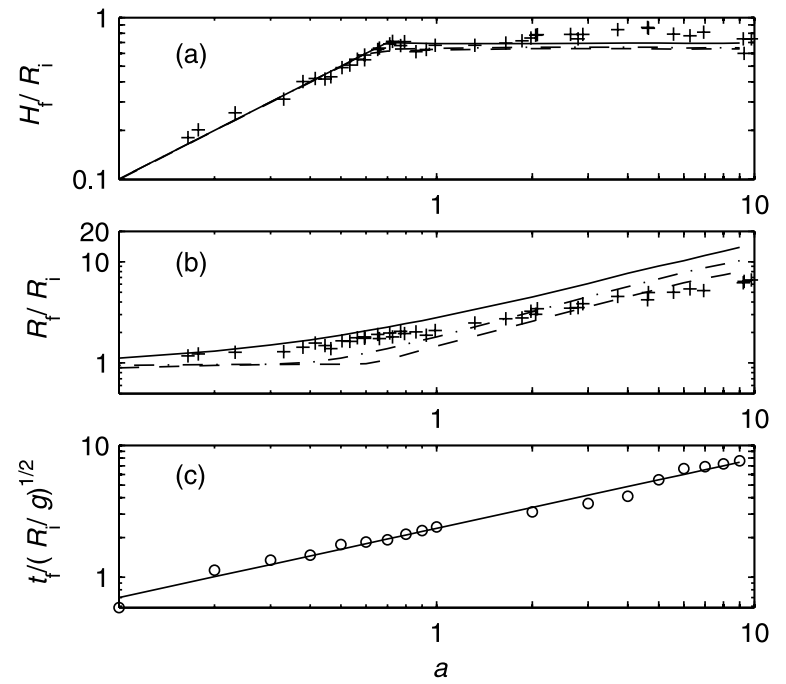

Figure 9. Normalized (a) final height of the deposit $H_{f} / R_{i}$, (b) final radius of the deposit $R_{f} / R_{i}$, and (c) time at which the front stops $t_{f} / \sqrt{R_{i} / g}$ as a function of the aspect ratio $a$. The crosses plotted on Figures $9 \mathrm{a}$ and $9 \mathrm{~b}$ correspond to the experiments performed by Lajeunesse et al. [2004]. The solid lines correspond to the numerical results obtained for cylindrical initial conditions (solid lines), paraboloidal initial conditions (dash-dotted lines) and conical initial condition (dashed lines).

numerically but appears already for $a=0.8$ as shown in Figure 8. Height profiles obtained for very large values of $a$, e.g., $a=10$, can be considered as a limiting envelope for all the profiles obtained for smaller values of $a$. For $a>0.7$, a conical profile is observed with roughly the same shape around the summit as the envelope profile, while the front zones clearly differ. The inflection point appears at the location of this divergence. As $a$ increases, the inflection point becomes more and more pronounced up to $a \sim 3$. For higher values, the morphology of the deposit becomes smoother and the inflection point finally disappears. Although the final deposit, for large value of $a$, is different from the laboratory deposit, the qualitative shape is similar with large and almost flat outer regions surrounding a steep central cone. In contrast to the laboratory results, the angle at the vicinity of the front never saturates toward a value of $5^{\circ}$, but instead decreases as $a$ increases. Moreover, the angle near the summit saturates for $a=6$ toward a value of $15^{\circ}-$ $18^{\circ}$. However, it must be kept in mind that uncertainties increase for high initial aspect ratios $a$ both in the numerical and laboratory experiments. Numerical experiments have also been performed with paraboloidal or conical initial shape conditions (Figures $8 \mathrm{~b}$ abd $8 \mathrm{c}$ ). In these experiments, a limiting profile envelope is not clearly observed.

\subsection{Deposit Parameters as a Function of $a$}

[51] Since the normalized deposit morphology is shown to depend only on $a$, simple empirical relations can be found relating morphological variables, e.g., the runout distance, the maximum height of the deposit, etc., to the aspect ratio [Lajeunesse et al. 2004; Lube et al., 2004]. Such relations can be investigated numerically by calculating for each numerical experiment the final highest height of the deposit
$H_{f}$, the final radius $R_{f}$, i.e., the front position, and the time $t_{f}$ at which the front stops. The normalized height $H_{f} / R_{i}$ of the deposit is shown in Figure 9a as a function of $a$. Clearly, for $a<0.7$, the normalized height increases linearly with $a$, as in the laboratory experiments, a direct consequence of the fact that $H_{f}=H_{i}$ for a truncated-cone-like deposit. For $a>$ $0.7, H_{f} / R_{i}$ saturates toward a value of the order of 0.7 as observed in Figure 8a.

[52] A striking result of these numerical experiments is their ability to reproduce the value $H_{f} / R_{i}=0.7$ related to the tangent of a critical angle $\delta_{c} \simeq 35^{\circ}$. The physical interpretation of this angle remains open to question. This angle was associated with an active Coulomb-yielding process within the granular column upon removal of the tube and to a related failure surface of angle $\delta_{c}$. Since our numerical model is based on an vertically averaged continuum description, which cannot take into account such a heterogeneous mode of deformation, the validity of this interpretation may be questioned. The dependence of this angle on the initial geometry of the released mass has been investigated numerically by considering both paraboloidal and conical initial shape conditions. The resultant angle values are shown in Figure 9 and depend on the initial geometry. The saturation value of $H_{f} / R_{i}$ is found to be $H_{f} / R_{i}=0.65$ for an initial paraboloidal shape, and $H_{f} / R_{i}=$ 0.64 for an initial conical shape, corresponding to angles $\delta_{p} \simeq 33^{\circ}$ and $\delta_{t} \simeq 32.5^{\circ}$, respectively. The angle $\delta_{t}$ is found to be close to the effective friction angle $\delta$ used in the numerical model. Indeed, in the model, a conical mass should remain at rest as long as its slope remains lower than $\tan \left(32^{\circ}\right) \simeq 0.62$ (see Figure 8c), as discussed in section 3.1. It would be therefore possible to roughly calibrate the effective friction angle $\delta$ of the numerical models by simply measuring the final normalized height of the deposit resulting from the spreading of a conical granular mass of known initial basal radius. Such a calibration procedure would be much simpler than performing laboratory experiments to measure the limiting angle of stability of a granular cone over a given substratum. However, such a procedure is not appropriate if the formalism proposed by Savage and Hutter [1989], involving $k_{\text {actpass, }}$, is used. In this case, the equation of equilibrium of the cone is more difficult to solve and involves now two parameters $\phi_{\text {bed }}$ and $\phi_{\text {int }}$.

[53] The final radius $R_{f}$ of the spreading mass is determined by the extent of the granular mass with a height higher than a lower cutoff of two bead diameters. The normalized runout distance, measured by $R_{f} / R_{i}$, is also in good agreement with the experiments up to a value $a \sim 1$. For larger values of $a$, the numerical simulation overestimates the runout distance. As discussed above, for such large values of the aspect ratio, a substantial part of the energy is converted into vertical kinetic energy, a feature that precludes the LWA. The runout distance is more sensitive to the initial shape of the released mass (Figure 9b). The normalized runout $R_{f} / R_{i}$ is obviously smaller for a paraboloidal shape and even much smaller for a conical shape due to the lower initial potential energy of the granular mass for a given $R_{i}$. As shown in Figure 9b, for an initial conical shape the granular mass begins to spread, i.e., $R_{f} f R_{i}>1$, for $a \geq 0.64$. The normalized final extent $R_{f} / R_{i}$ decreases with increasing $\delta$ for a given $a$. While $R_{f} / R_{i}$ decreases continuously for $a=0.9\left(20^{\circ} \leq \delta \leq 30^{\circ}\right)$, it 

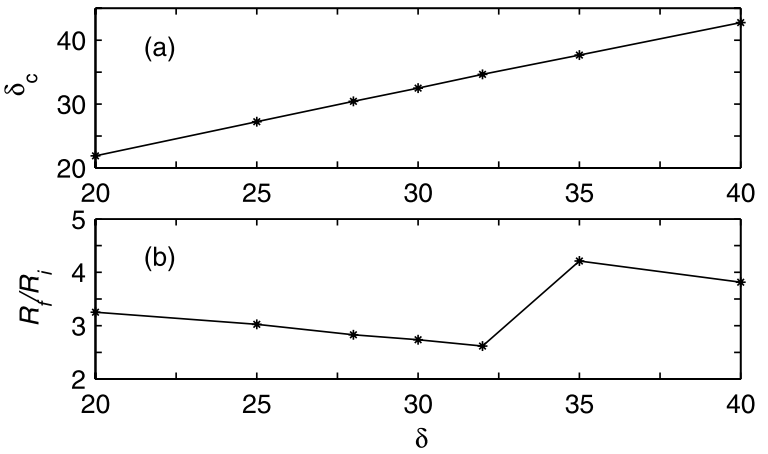

Figure 10. (a) Plot of $\delta_{c}=\arctan \left(H_{f} / R_{i}\right)$ in degrees and (b) normalized radius $R_{f} / R_{i}$ of the deposit as a function of the friction angle $\delta$ used in the numerical model. The points calculated with $20^{\circ} \leq \delta \leq 30^{\circ}$ are for $a=0.9$, and the points calculated with $35^{\circ} \leq \delta \leq 40^{\circ}$ are for $a=2$.

increases for a value of $a=2$ from $\delta=35^{\circ}$ (Figure 10). For $a=$ $2\left(35^{\circ} \leq \delta \leq 40^{\circ}\right)$, the normalized extent is found to decrease more quickly than for $a=0.9$. The numerical deposit depends on the friction angle in contrast with the conclusion of the experimental study performed by Lube et al. [2004]. For small values of $a$, the normalized runout is found to be only slightly dependent on the friction angle, as shown in Figure 2. This sensitivity increases with increasing $a$.

[54] The arrest time is difficult to determine due to the arrest phase complexity (Figure 4). The arrest time of the front has been chosen as the time at which the front of the granular mass with height greater than two grains diameter stops. The calculated arrest times are smaller but in the range of those measured by Lube et al. [2004], even though the details of the arrest phase are different as previously mentioned in section 4.2. The numerical fit, represented in solid lines on Figure 9c, leads to $\tilde{t}_{f}=2.35$ $a^{0.51} \sim 2.35 \sqrt{a}$ which then gives

$$
t_{f} \sim 2.5 \sqrt{\frac{H_{i}}{g}} .
$$

Lube et al. [2004] also found a relation involving the square root of $a$ but with a proportionality factor of the order of 3 . The calculated arrest time of the front is slightly less than twice the free fall time, defined as the time of fall for a particle initially at a height $H_{i}$. Note that the scaling relation between the arrest time and the square root of $a$ is very well reproduced by the model even for high values of $a$, and that $t_{f}$ does not depend on the initial radius of the cylinder. It can be compared here with the arrest time of the front derived from the analytical solution of Mangeney et al. [2000] for the 1-D semi-infinite dam break problem. In this solution, the position of the front is given by

$$
x_{f}=2 c_{0} t-\frac{1}{2} g \tan \delta t^{2},
$$

where $c_{0}=\sqrt{g H_{i}}$ is the gravity wave celerity. The front velocity is then

$$
v_{f}=2 c_{0}-g \tan \delta t .
$$

The front stops when the velocity decelerates to zero due to friction:

$$
t_{f}^{\prime}=\frac{2 c_{0}}{g \tan \delta}=\frac{2}{\tan \delta} \sqrt{\frac{H_{i}}{g}} .
$$

In this 1-D case, the time at which the front stops is given by $t_{f}^{\prime} \sim 3.2 \sqrt{H_{i} / g}$. The coefficient value 3.2 is larger than the value of 2.5 found in the 2-D case studied here. The difference may be due to an enhanced effect of the front velocity deceleration due to radial spreading. In all cases, this must be linked to the friction coefficient $\mu=\tan \delta$ as for the saturation of $H_{f} / R_{i}$.

\subsection{Effective Friction $\mathrm{H} / \mathrm{L}$}

[55] In geological literature, characterization of geological gravitational events very often make uses of the notion of effective friction $\mu_{e}=H / L$, where $H$ is the fall height and $L$ the horizontal runout distance [e.g., Siebert et al., 1987; McEwen, 1989; Stoopes and Sheridan, 1992]. The small values of $\mu_{e}$ deduced from geological observations are generally interpreted as evidence of a low apparent coefficient of friction implying small friction angles in numerical models. Moreover a physical interpretation of the observed apparent decrease of the effective friction in the case of high volume events is still open to question (see Legros [2002] for a review). Within this study, the effective friction may be defined as the ratio $H_{i} / R_{f}$. Both experiments and numerical simulations show that $\mu_{e}$ increases with $a$ (Figure 11). A numerical fit of the experimental data shows that $\mu_{e}$ is proportional to the square root of the aspect ratio. Such a scaling relationship can be directly derived from the dimensional analysis of the proposed minimal model where the effective friction is defined as

$$
\mu_{e}=\frac{H_{i}}{R_{f}}=\frac{a}{\tilde{R}_{f}} .
$$

As in the work by Lajeunesse et al. [2004], the deposit for $a>0.7$ can be approximated by a cone of volume $V_{f}=$ $\pi / 3 H_{f} R_{f}^{2}$. Making use of volume conservation and of the observed saturation of $H_{f}$ toward the limiting value $H_{f}=$ 0.7 , the normalized radius is given by

$$
a>0.7, \tilde{R}_{f}=\sqrt{\frac{3 a}{0.7}},
$$

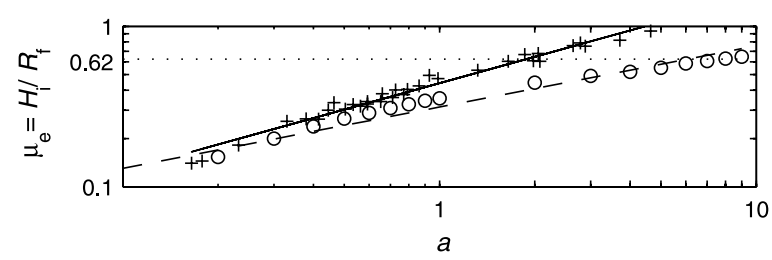

Figure 11. Effective friction $\mu_{e}=H_{i} / R_{f}$ as a function of the aspect ratio $a$ obtained from numerical simulation (circles) and from experiments (crosses). Best fit lines for the experimental data for an initial cone (solid lines) and the numerical experiments (dashed lines) have been added. The value of the friction coefficient $\mu=\tan \left(32^{\circ}\right)=0.62$ is represented by a dotted line. 


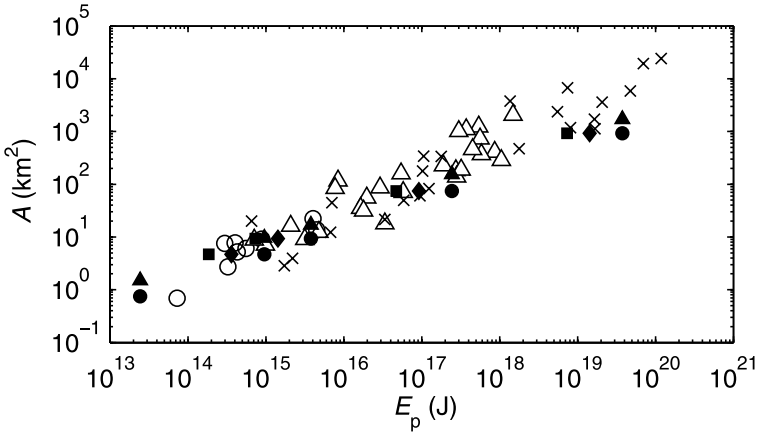

Figure 12. Area $A$ covered by the deposit of avalanches or rockfall as a function of the initial potential energy. Geological data are from Dade and Huppert [1998]: white circles correspond to nonvolcanic events, white triangles correspond to volcanic events, and crosses correspond to extraterrestrial events. Numerical simulations with $\delta=32^{\circ}$ (solid circles) and with $\delta=20^{\circ}$ (solid triangles) for the Earth, and $\delta=32^{\circ}$ for Mars (solid diamonds) and for the Moon (solid squares) have been added.

leading to

$$
\mu_{e} \sim 0.5 \sqrt{a} .
$$

Numerically, a scaling relation $\mu_{e} \propto a^{0.52}$ is found for $a<1$ with an uncertainty in the exponent of $0.52 \pm 0.02$, which is close to the experimented square-root type of scaling. This is consistent with an interpretation in terms of two regimes. For small aspect ratios, only a few parts of the granular material are actually in motion, leading to a weak global dissipation. As $a$ increases, larger amounts of material are mobilized and the energy dissipation increases. This is consistent with the experimental results shown by Lajeunesse et al. [2004] which show the dissipation of only a fraction of the initial potential energy in the first regime while, in the second regime, dissipation of almost the whole initial gravitational energy is observed. Geologically the height of the released mass is limited by the height of the relief. For extremely large volumes, an increase in the mass involved in the destabilization is then expected to result from a lower aspect ratio, leading to a lower effective friction $\mu_{e}$ (Figure 11). This may possibly explain why very large masses have greater mobility depending on the actual volumes involved.

[56] The overall numerical experiments show that, in the domain of validity of the LWA, i.e., $a<1$, the effective friction is lower than the coefficient of friction actually used in the model, i.e., $\mu=\tan \left(32^{\circ}\right)=0.62$. In fact, it is clear from Figure 11 that the effective friction does not correspond to the empirical friction angle $\delta$.

\section{Comparison With Geological Data}

[57] A strong correlation between the area overrun by the avalanche and the potential energy of the avalanche has been shown by Dade and Huppert [1998] on the basis of the analysis of geological events associated with cataclysmic failure of volcanic and nonvolcanic slopes on the Earth, the Moon, and Mars (Figure 12). Extraterrestrial events follow the same trend as terrestrial events. The best fit leads to a power law relation with an exponent in the range $0.58-0.68$ (the 95\% confidence interval) [Dade and Huppert, 1998].

[58] When using the dimensionless generic solution (8), (9), and (10), the final height, runout and time of deposit of a granular mass, characterized by its initial aspect ratio $a$ and radius $R_{i}$, can be deduced from the scaling relation displayed in Figure 9. Note that such a scaling relation is also meaningful for extraterrestrial collapses, due to the invariance of the dimensionless solution with respect to acceleration due to gravity $g$. While such an invariance leads to the same deposit area for terrestrial and extraterrestrial events, the potential energy does depend on $g$. This makes it possible to report (Figure 12) numerical results, in terms of the deposit area and initial potential energy, with the observations of Dade and Huppert [1998]. Acceleration due to gravity was taken to be $g=9.81 \mathrm{~ms}^{-2}, g=3.7 \mathrm{~ms}^{-2}$, $g=1.6 \mathrm{~ms}^{-2}$ for the Earth, Mars and the Moon, respectively. These new numerical points are found to follow the same trend as the geological data both for terrestrial and telluric conditions despite the fact that our minimal model may appear rather simplistic with regard to the complexity involved in the natural events. Note that here the friction angle $\delta=32^{\circ}$ has been kept constant with no attempt to fit the actual data. When using $\delta=20^{\circ}$, a more extended area is obtained (black triangles in Figure 12) which also falls in the range of the observed area. It is possible to represent analytically the observed correlation between the avalanche area and its initial potential energy.

[59] The initial potential energy of a cylinder of radius $R_{i}$ and height $H_{i}$ is given by

$$
E_{p}=\frac{M g H_{i}}{2}=\phi \rho g \pi \frac{a^{2}}{2} R_{i}^{4},
$$

where $\rho \sim 2500 \mathrm{~kg}$ and $\phi \sim 0.62$ in the experiments, while the aerial extent is given by

$$
A=\pi R_{f}^{2}=\pi R_{i}^{2} \tilde{R}_{f}^{2} .
$$

After elimination of $R_{i}$, the scaling relation between $E_{p}$ and $A$ is readily obtained as

$$
E_{p}=\frac{\phi \rho a^{2}}{2 \pi \tilde{R}_{f}^{4}(a)} A^{2}=f(a) A^{2}
$$

and $E_{p}$ scales with the square of $A$ with a prefactor that depends only on $a$. Introducing equation (37) into equation (41), leads to

$$
f(a) \sim \frac{\phi \rho g(0.7)^{2}}{18 \pi},
$$

which is actually independent of $a$. This analysis leads to power law scaling relation between the avalanche area $A$ and the potential energy with an exponent of $1 / 2$, slightly smaller than the minimum exponent 0.58 estimated from observational data. Such a difference may result from the two-dimensional geometry of the experimental spreading while geological avalanches may be regarded as essentially a 1-D flow. Although the function $f(a)$ does depend on 
acceleration due to gravity $g$, data obtained from terrestrial and extraterrestrial events seem in Figure 11 to collapse. However, the actual observations do not provide sufficient resolution to single out the trend for a particular planet or a $g$ effect.

\section{Conclusion}

[60] In this paper we propose a minimal model based on a long-wave kinematic approximation and a simple Coulomb type effective friction. The dimensionless analysis of the governing equations (1)-(4) of the model is consistent with the experimental observation that the dynamics of spreading depend on the initial aspect ratio $a$ but not on the initial mass, and the dimensionless equations do not depend on acceleration due to gravity.

[61] Numerical experiments, using such a minimal model, are in good agreement with the laboratory experiments of Lajeunesse et al. [2004] on the spreading of granular cylinders on a horizontal plane, at least for regimes corresponding to an initial aspect ratio $a \leq 1$. In such a regime, numerical experiments correctly reproduce laboratory experiments during dynamic spreading up to the arrest phase, and the dynamics seems to depend only weakly on the effective friction coefficient, i.e., the friction angle, at least in the range of values explored by Lube et al. [2004]. In the same regime, the final shape of the deposit is also found to depend weakly on the friction coefficient. This sensitivity increases with $a$.

[62] Numerical experiments confirm the existence of two dynamic spreading regimes depending on the initial aspect ratio $a$, as observed experimentally. The saturation of the normalized height of the deposit $H_{f} / R_{i}$, as a function of the normalized radius $r / R_{i}$, observed experimentally is accurately reproduced by the model. For an initial conical shape, this saturated value is found to be close to the effective friction coefficient. This suggests the need for new experiments to calibrate the angle of effective friction of the proposed minimal model, which could be identified as the angle of stability of a conical mass suddenly released from rest.

[63] The good agreement observed between numerical and laboratory experiments for $a<1$ strongly suggests that in this case, the gravitational spreading involves a flow regime characterized by vertical velocity fluctuations which can be averaged out over a vertical length smaller or equal to the actual height of the granular material, and that vertical acceleration remains small compared to the vertical pressure gradient. However, such an agreement becomes more qualitative in the second dynamic regime characterized by large values of the initial aspect ratio $a>1$. Note also that for this regime, the final shape of the deposit becomes relatively sensitive to the effective friction coefficient. Moreover in both regimes, the experimentally observed arrest phase and the late relaxation process are not reproduced by the minimal model used in this study. This suggest that the minimal model should be extended to provide a more realistic rheological behavior. The laboratory experiments suggest that both propagation and consolidation phases are involved in the spreading of the granular mass. In our minimal formulation, the model is expressed in terms of total stresses. This approximation is only valid, for drained or undrained behavior, when the consolidation timescale is much larger than the propagation time. This is clearly not the case during the arrest phase and the late relaxation process, suggesting that pore pressure dissipation and effective stresses should be taken into account. The model could be extended to include the coupling between a solid skeleton and pore fluids, via an approach similar to mixture theories [Pastor et al., 2002; Iverson and Denlinger, 2001], in order to take vertical consolidation and pore pressure decrease into account.

[64] The scaling relations of $\tilde{H}_{f}(a)$ and $\tilde{R}_{f}(a)$, suggested by laboratory and numerical experiments, provide a useful way of estimating the overun area $A$ for gravitation collapse events both in the case of terrestrial and extraterrestrial conditions. Moreover, these experiments show that the time needed for the front to stop scales directly with the characteristic time of the free fall divided by the effective coefficient of friction $\mu=\tan \delta$, and can be recovered from the $\tilde{t}_{s}(a)$ scaling. All the numerical results are shown to fall within the range of the data from deposits of natural events despite the simplicity of the numerical configuration. All these results suggest that classical interpretation of the effective friction coefficient in terms of the ratio $H / L$, generally advocated for the mobility of geological events, is very questionable since this ratio is shown to be dependent on the aspect ratio of the initial released mass. Such a dependence may provide an alternative interpretation of the high mobility of natural events involving the destabilization of large volumes of soil material.

[65] Acknowledgments. We thank M. O. Bristeau and B. Perthame for fruitful discussions. This work was supported by the Action Concertee Incitative Nouvelles Interfaces des Mathématiques (CNRS) and Action Thematique Incitative Prioritaire (CNRS). IPGP contribution 2075.

\section{References}

Aranson, I. S., and L. S. Tsimring (2002), Continuum theory of partially fluidized granular flows, Phys. Rev. E, 65, 061303.

Audusse, E., F. Bouchut, M. O. Bristeau, R. Klein, and B. Perthame (2004), A fast and stable well-balanced scheme with hydrostatic reconstruction for shallow water flows, SIAM J. Sci. Comput., 25(6), 2050-2065.

Bouchut, F. (2004), Nonlinear Stability of Finite Volume Methods for Hyperbolic Conservation Laws and Well-Balanced Schemes for Sources, Frontiers Math. Ser, vol. VIII, Springer, New York.

Dade, W. B., and H. E. Huppert (1998), Long runout rockfalls, Geology, 26, $803-806$.

Daerr, A., and S. Douady (1999), Sensitivity of granular surface flows to preparation, Europhys. Lett., 47, 324-330.

Denlinger, R. P., and R. M. Iverson (2001), Flow of variably fluidized granular masses across three-dimensional terrain: 2 . Numerical predictions and experimental tests, J. Geophys. Res., 106(B1), 553-566.

Denlinger, R. P., and R. M. Iverson (2004), Granular avalanches across irregular three-dimensional terrain: 1 . Theory and computation, J. Geophys. Res., 109, F01014, doi:10.1029/2003JF000085.

Douady, S., B. Andreotti, and A. Daerr (1999), On granular surface flow equations, Eur. Phys. J. B., 11, 131-142.

Ertas, D., G. S. Grest, T. C. Halsey, D. Levine, and L. E. Silbert (2001), Gravity-driven dense granular flows, Europhys. Lett., 56(2), 214-220.

Felix, G., and N. Thomas (2004), Relation between dry granular flow regimes and morphology of the deposits: Formation of levées in pyroclastic deposits, Earth Planet. Sci. Lett., 221(1-4), 197-213.

Gray, J. M. N. T., M. Wieland, and K. Hutter (1999), Gravity driven free surface flow of granular avalanches over complex basal topography, Proc. R. Soc. London, Ser. A, 455, $1841-1874$

Hutter, K., T. Koch, C. Pluss, and S. B. Savage (1995), The dynamics of avalanches of granular materials from initiation to runout. part II. Experiments, Acta Mech., 109, 127-165.

Iverson, R. M. (1997), The physics of debris flows, Rev. Geophys., 35(3), 245-296. 
Iverson, R. M., and R. P. Denlinger (2001), Flow of variably fluidized granular masses across three-dimensional terrain: 1 . Coulomb mixture theory, J. Geophys. Res., 106(B1), 537-552.

Iverson, R. M., M. Logan, and R. P. Denlinger (2004), Granular avalanches across irregular three-dimensional terrain: 2. Experimental tests, J. Geophys. Res., 109, F01015, doi:10.1029/2003JF000084

Kerswell, R. R. (2005), Dam break with Coulomb friction: A model of granular slumping?, Phys. Fluids, 17, 057101.

Lajeunesse, E., A. Mangeney-Castelnau, and J. P. Vilotte (2004), Spreading of a granular mass on a horizontal plane, Phys. Fluids, 16, 2371-2381.

Legros, F. (2002), The mobility of long-runout landslides, Eng. Geol., 63, $301-331$

Lube, G., S. Sparks, and M. Hallworth (2003), Axisymmetric granular collapse, paper presented at Geophysical Granular and Particle-Laden Flows Workshop, Isaac Newton Inst., Bristol, U. K., 27-31 Oct.

Lube, G., H. E. Huppert, R. S. J. Sparks, and M. A. Hallworth (2004), Axisymmetric collapses of granular columns, J. Fluid Mech., 508, 175199.

Mangeney, A., and F. Califano (1998), The shallow-ice approximation for anisotropic ice: Formulation and limits, J. Geophys. Res., 103(B1), $691-$ 705.

Mangeney, A., P. Heinrich, and R. Roche (2000), Analytical solution for testing debris avalanche numerical models, Pure Appl. Geophys., 157, $1081-1096$.

Mangeney-Castelnau, A., J.-P. Vilotte, M. O. Bristeau, B. Perthame, F. Bouchut, C. Simeoni, and S. Yerneni (2003), Numerical modeling of avalanches based on Saint Venant equations using a kinetic scheme, J. Geophys. Res., 108(B11), 2527, doi:10.1029/2002JB002024.

McEwen, A. S. (1989), Mobility of large rock avalanches: Evidence from Valles Marineris, Mars, Geology, 17, 1111-1114.

Müller, D. (1995), Techniques informatiques efficaces pour la simulation de milieux granulaires par des méthodes d'éléments distincts, Ph.D. thesis, Ecole Polytech. Féd. de Lausanne, Lausanne, France.

Naaim, M., S. Vial, and R. Couture (1997), Saint-Venant approach for rock avalanches modelling, paper presented at Saint Venant Symposium, Ecole Nat. des Ponts et Chaussees, Paris, 28-29 Aug.

Pastor, M., M. Quecedo, J. A. F. Merodo, M. I. Herreros, E. Gonzales, and P. Mira (2002), Modelling tailings dams and mine waste dumps failures, Geotechnique, 52(8), 579-591.

Pitman, E. B., C. C. Nichita, A. K. Patra, A. C. Bauer, M. Sheridan, and M. Bursik (2003), Computing granular avalanches and landslides, Phys. Fluids, 15, 3638-3646.
Pouliquen, O. (1999), Scaling laws in granular flows down rough inclined planes, Phys. Fluids, 11, 542-548.

Pouliquen, O., and Y. Forterre (2002), Friction law for dense granular flows: Application to the motion of a mass down a rough inclined plane, J. Fluid Mech., 453, 133-151.

Savage, S. B., and K. Hutter (1989), The motion of a finite mass of granular material down a rough incline, J. Fluid Mech., 199, 177-215.

Sheridan, M. F., A. J. Stinton, A. Patra, E. B. Pitman, A. Bauer, and C. C. Nichita (2005), Evaluating Titan2D mass-flow model using the 1963 Little Tahoma Peak avalanches, Mount Rainier, Washington, J. Volcanol. Geotherm. Res., 139, 89-102.

Siebert, L., H. Glicken, and T. Ui (1987), Volcanic hazards from Bezymiannyand Bandai-type eruptions, Volcanol. Bull., 49, 435-459.

Staron, L., and E. J. Hinch (2004), Study of the collapse and spreading of a granular mass using discrete numerical simulation, Eos Trans. $A G U$, 85(47), Fall Meet. Suppl., Abstract H33G-07.

Staron, L., J. P. Vilotte, and F. Radjai (2002), Pre-avalanche instabilities in a tilted granular pile, Phys. Rev. Lett., 89, 204-302.

Stoopes, G. R., and M. F. Sheridan (1992), Giant debris avalanches from the Colina Volcanic complex, Mexico: Implications for long-runout landslides $(>100 \mathrm{~km})$ and hazard assessment, Geology, 20, 299-302.

Volfson, D., L. S. Tsimring, and I. S. Aranson (2003), Partially fluidized shear granular flows: Continuum theory and molecular dynamics simulations, Phys. Rev. E, 68, 021301.

Wieland, M., J. M. N. T. Gray, and K. Hutter (1999), Channelized free surface flow of cohesionless granular avalanches in a chute with shallow lateral curvature, J. Fluid Mech., 392, 73-100.

A. Aubertin, A. Mangeney-Castelnau, M. Pirulli, and J. P. Vilotte, Département de Sismologie, Institut de Physique du Globe de Paris, 4 place Jussieu, 75252 Paris cedex 05, France. (mangeney@ipgp.jussieu.fr; vilotte@ipgp.jussieu.fr)

F. Bouchut, Département de Mathématique et Applications, Ecole Normale Supérieure, 45 rue d'Ulm, F-75230 Paris cedex 05, France. (fbouchut@dma.ens.fr)

E. Lajeunesse, Laboratoire de Dynamique des Systèmes Géologiques, Groupe de Recherches sur l'Erosion et les Eaux Continentales, Institut de Physique du Globe de Paris, 4 place Jussieu, F-75252 Paris cedex 05, France. (lajeunes@ipgp.jussieu.fr)

M. Pirulli, Politcnico, Torino, Italy. (marina.pirulli@polito.it) 